\title{
Classical gravity from loop amplitudes
}

\author{
N. Emil J. Bjerrum-Bohr $\odot,{ }^{1,{ }^{*}}$ Poul H. Damgaard, ${ }^{1}$ Ludovic Planté, ${ }^{2, \dagger}$ and Pierre Vanhove $\odot^{3,4,5, \$}$ \\ ${ }^{1}$ Niels Bohr International Academy, Niels Bohr Institute, University of Copenhagen, \\ Blegdamsvej 17, DK-2100 Copenhagen, Denmark \\ ${ }^{2} 20$ rue Marie et Pierre Curie, 92110 Clichy, France \\ ${ }^{3}$ Institut de Physique Theorique, Université Paris-Saclay, CEA, CNRS, \\ F-91191 Gif-sur-Yvette Cedex, France \\ ${ }^{4}$ National Research University Higher School of Economics, \\ 20 Myasnitskaya ulitsa, Moscow 101000, Russia \\ ${ }^{5}$ Theoretical Physics Department, CERN, 1211 Geneva 23, Switzerland
}

(Received 2 May 2021; accepted 28 May 2021; published 20 July 2021)

\begin{abstract}
We describe an efficient method for extracting the parts of $D$-dimensional loop integrals that are needed to derive observables in classical general relativity from scattering amplitudes. Our approach simplifies the soft-region method of integration by judiciously combining terms before the final integrations. We demonstrate the method by computing the required integrals for black-hole scattering to the second post-Minkowskian order in Einstein gravity coupled to scalars. We also confirm recent results at the third post-Minkowskian order regarding universality and high-energy behavior of gravitational interactions in maximal supergravity.
\end{abstract}

DOI: 10.1103/PhysRevD.104.026009

\section{INTRODUCTION}

The detection of gravitational waves from binary mergers $[1,2]$ has opened a new and exciting avenue for testing Einstein's theory of gravity at extreme energies and at relativistic velocities. Although detailed comparisons of gravitational wave signals eventually require numerical general relativity, there is an urgent need for highly precise analytical computations as well since these are what is fed into accurate catalogs of gravitational wave templates. A particularly promising new approach for such analytical calculations is based on the so-called post-Minkowskian expansion in general relativity [3-6]. This is the specialrelativistic regime of general relativity where relative velocities of two gravitationally interacting bodies are not assumed to be much smaller than the speed of light. As such, this situation is ideally suited for the relativistic quantum field theory description of gravity when truncated to the classical sector. The $S$ matrix describing the gravitational scattering of two massive objects from Minkowskian infinity to Minkowskian infinity will indeed

\footnotetext{
*bjbohr@nbi.dk

†udo.plan@hotmail.fr

*pierre.vanhove@ipht.fr
}

Published by the American Physical Society under the terms of the Creative Commons Attribution 4.0 International license. Further distribution of this work must maintain attribution to the author(s) and the published article's title, journal citation, and DOI. Funded by SCOAP. automatically include all orders in velocity to any given order in Newton's constant $G_{N}$. When restricted to the classical part of scattering this should coincide with the post-Minkowskian expansion of Einstein gravity.

There are numerous subtleties associated with this new and promising approach that need to be understood. A folk theorem of relativistic quantum field theory states that the only basic observables are $S$-matrix elements and this seems a perfect starting point for a scattering calculation for the classical limit. In practice, the $S$ matrix needs to be defined carefully in the presence of massless fields and one could worry that such quantum field theoretic subtleties could translate into effects that survive when taking the classical limit. Another concern could be the application the fieldtheoretic method to gravity because of its coupling to the energy-momentum tensor. Is the high-energy limit of gravitational scattering where Mandelstam variable $s$ is much bigger than the sum of the two masses squared $\left(m_{1}+m_{2}\right)^{2}$ then equal to massless scattering? At one-loop order, this is the case [5]. At two-loop order, the calculations of [7-10] does not lead to that conclusion, and indeed the scattering angle from that two-loop order computation, when extrapolated to very high energies, diverges. Recently, it has been shown [11] that this disconcerting conclusion is resolved when the classical parts of the scattering amplitude are extracted from the so-called soft region of the loop integrals, rather than from what is known as the potential region, which is the natural starting point of a low-energy computation.

This situation has called for a renewed focus on obtaining a more systematic framework in which to compute those 
parts of gravitational loop diagrams that will contribute to classical scattering in general relativity. In this paper, we will introduce a formalism that we will argue simplifies computations based on the soft-regions method [11-13]. While the purpose of the method is to identify those terms that contribute to classical scattering $[5,14]$, the formalism is also immediately applicable to both the pieces in the amplitude that are more singular than the classical one in the $\hbar \rightarrow 0$ limit and to those that correspond to quantum mechanical corrections, which are subleading in the $\hbar \rightarrow 0$ limit. A main advantage of our method is that it significantly reduces the number of master integrals that need to be computed. This is already an important simplification at two-loop order and it will be of even more value at higherloop order. Our independent calculation at two-loop order for $\mathcal{N}=8$ supergravity confirms the recent results of $[11,13,15,16]$.

Before going into the technical details of our calculation, we find it useful to highlight the origin of the additional classical terms of the soft region as compared to those of the potential region. Intuitively, the post-Minkowskian expansion can appear as a natural resummation of terms of the post-Newtonian expansion. In the free-particle massive propagator, very schematically, and ignoring the split into energy and three-momentum,

$$
\frac{1}{k^{2}-m^{2}} \sim \frac{1}{k^{2}}\left(1+\frac{m^{2}}{k^{2}}+\left(\frac{m^{2}}{k^{2}}\right)^{2}+\cdots\right),
$$

and the potential-region integration indeed resums such contributions correctly at one-loop order. However, this one-loop result is already quite nontrivial and it does not seem to carry over to higher orders. The reason is that inside the loop integrals, what plays the role of mass $m$ will be combinations of momenta. At specific regions of the integrations what effectively acts as a mass term in the propagator can therefore vanish. At these points, dimensional regularization, if used in conjunction with the above expansion, will naïvely put these terms to zero for the same reason that the massive tadpole,

$$
\int \frac{d^{4-2 \epsilon} k}{(2 \pi)^{4-2 \epsilon}} \frac{1}{k^{2}-m^{2}+i \varepsilon}=-i \pi^{2-\epsilon} \frac{\Gamma(\epsilon-1)}{\left(m^{2}\right)^{\epsilon-1}},
$$

will be set, incorrectly, to zero in dimensional regularization if one integrates order by order in the small-mass expansion of Eq. (1). For $m^{2} \sim \sigma^{2}-1$ such a tadpole integral has a $1 /\left(\sigma^{2}-1\right)^{\epsilon}$ dependence which is the origin of the radiationreaction contributions. A particular consequence of the noncommutativity between the integration and the small velocity expansion results in the vanishing of the master integrals evaluated in Sec. VE 3 if one integrates the termby-term expansion. Consequently, all the radiation-reaction contributions to the two-loop classical contribution vanish.
Concretely, the classical part of the two-loop amplitude gets a contribution from the master integral $\mathcal{I}_{6}(\sigma)$ which evaluates to

$$
\begin{aligned}
\mathcal{I}_{6}(\sigma)= & \frac{\left(4 \pi e^{-\gamma_{E}}\right)^{2 \epsilon} \epsilon^{3}}{8 \pi^{3}} \operatorname{arcsinh}\left(\sqrt{\frac{\sigma-1}{2}}\right) \\
& \times\left(\pi+2 i\left(\frac{-1}{4\left(\sigma^{2}-1\right)}\right)^{\epsilon} \operatorname{arcsinh}\left(\sqrt{\frac{\sigma-1}{2}}\right)\right) \\
& +\mathcal{O}\left(\epsilon^{4}\right),
\end{aligned}
$$

where $\sigma=p_{1} \cdot p_{2} /\left(m_{1} m_{2}\right)$ with $p_{i}$ as the momenta of the incoming massive scalars of mass $m_{i}$, and $\epsilon=(4-D) / 2$ is the dimensional regularization parameter. This integral, like all the master two-loop integrals, has a regular static limit $\sigma \rightarrow 1$ only for $\epsilon$ negative as required for the infrared behavior (see for instance [17]).

Performing the series expansion in $\epsilon$ near 0 (and negative) with $\sigma$ greater than 1 fixed gives

$$
\begin{aligned}
\underset{\epsilon \rightarrow 0^{-}}{\operatorname{series}} \mathcal{I}_{6}(\sigma)= & \frac{\left(4 \pi e^{-\gamma_{E}}\right)^{2 \epsilon} \epsilon^{3}}{8 \pi^{3}} \operatorname{arcsinh}\left(\sqrt{\frac{\sigma-1}{2}}\right) \\
& \times\left(\pi+2 i \operatorname{arcsinh}\left(\sqrt{\frac{\sigma-1}{2}}\right)\right)+\mathcal{O}\left(\epsilon^{4}\right),
\end{aligned}
$$

which matches the result in Eq. (D.3) of [8]. Whereas the series expansion first in $\sigma$ near 1 (and greater than 1) with $\epsilon$ small and negative fixed, gives

$$
\begin{aligned}
\underset{\sigma \rightarrow 1^{+}}{\operatorname{series}} \mathcal{I}_{6}(\sigma)= & \frac{\left(4 \pi e^{-\gamma_{E}}\right)^{2 \epsilon} \epsilon^{3}}{8 \pi^{2}} \operatorname{arcsinh}\left(\sqrt{\frac{\sigma-1}{2}}\right) \\
& +\mathcal{O}\left(\epsilon^{4}\right),
\end{aligned}
$$

which is the result in Eq. (4.111) of [12]. At this order in $\epsilon$ one notices that the result in (5) does not have the imaginary part present in (4). This shows the importance of keeping the contribution $\left(-1 /\left(4\left(\sigma^{2}-1\right)\right)\right)^{\epsilon}$ in order to obtain the radiation-reaction contributions to the classical part of the amplitude. And because $(-1)^{\epsilon}=1+i \pi \epsilon+O\left(\epsilon^{2}\right)$ this factor contributes to both the real and imaginary part of the classical terms of the amplitude. As we will show in Sec. III B 3 this factor will be instrumental in showing the relation between the real part and the infrared divergence of the imaginary part of the radiation-reaction contribution at two-loop order [15].

Of course, eventually, the result must be unambiguous, but the latter order of limits seems to require a different approach to determine correctly the integration constants of the set of differential equations. We will employ the former order of limit here, and use it to clarify very precisely the difference between the recent results of two different computations in [11,12]. The computation of [12] provides all the terms relevant for the amplitude in the potential region. We should stress that our purpose here is to focus only on how to extract all classical terms of scattering amplitudes at loop orders. 
At two-loop order the calculation very neatly separates so that the final answer for the amplitude is decomposed into all the terms from the potential region plus new contributions that arise from the above mechanism. Some of these new terms at two-loop order are real and some are both imaginary and divergent. These new terms were first identified in Refs. [11], and we confirm those results in all details. A surprising feature is that the new terms which are real, being of half-integer order when expanded at low energy, find no place within the conventional post-Newtonian expansion $[18,19]$ of the conservative part of the interaction Hamiltonian. These additional terms have been identified as radiation-reaction pieces $[11,15]$ that normally would have to be treated separately in the post-Newtonian expansion. A recent calculation of Damour [20] confirms this picture, also suggesting a shortcut towards identifying the terms that must be added to the potential-region calculation in Einstein gravity, and which will render the high-energy scattering angle finite at this order. It is interesting to speculate how the appearance of these new pieces may have repercussions at the conventional level of the post-Minkowskian expansion from three-loops and higher. ${ }^{1}$ A first calculation of the fourth postMinkowskian result for the conservative part of the interactions of scalar black holes has already appeared [21].

We outline the paper as follows. In Sec. II we review how to determine classical general relativity from scattering amplitudes. We will discuss how it is possible to expand the scattering amplitudes at second (in Sec. III A) and third (in Sec. III) post-Minkowskian order in terms of a set of classical basis integrals multiplied with coefficients determined from unitarity. In Sec. III we give the results of the evaluation of the third post-Minkowskian contribution in maximal supergravity. We show that the classical contribution to the amplitude is independent of the helicity configuration. We then compare the results with the ones from the potential and soft integration region. In Sec. IV we evaluate the double-box integral contributions to the maximal supergravity amplitude. In Sec. V we determine the basis of master integrals used in the evaluation of the two-loop double-box integrals. In the Appendix we explain how to reduce the generic two-loop double-box integrals to the two-loop master integral with a generalized propagator that we use in this computation. Finally, we will conclude and look ahead.

\section{GENERAL RELATIVITY FROM QUANTUM FIELD THEORY}

The Einstein-Hilbert Lagrangian minimally coupled to two massive scalar fields reads

$$
\begin{aligned}
\mathcal{L}_{\mathrm{EH}}= & \int d^{4} x \sqrt{-g}\left[\frac{R}{16 \pi G_{N}}+\frac{1}{2} g^{\mu \nu}\left(\partial_{\mu} \phi_{1} \partial_{\nu} \phi_{1}+\partial_{\mu} \phi_{2} \partial_{\nu} \phi_{2}\right)\right. \\
& \left.-m_{1}^{2} \phi_{1}^{2}-m_{2}^{2} \phi_{2}^{2}\right]
\end{aligned}
$$

in this equation $G_{N}$ denotes the Newton constant, $R$ defines the Ricci scalar, and $g$ is the determinant of the metric: $g_{\mu \nu}(x) \equiv \eta_{\mu \nu}+\sqrt{32 \pi G_{N}} h_{\mu \nu}(x)$ expanded around a Minkowski background, $\operatorname{diag} \eta_{\mu \nu} \equiv(1,-1,-1,-1)$. We consider scattering events organised in a perturbative expansion,

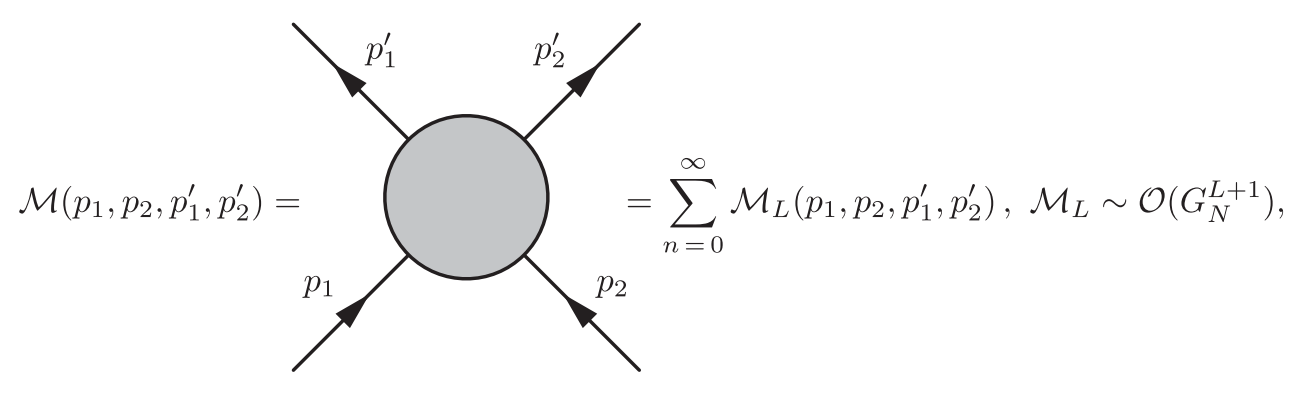

where $p_{1}$ and $p_{2}$ are incoming momenta and $p_{1}^{\prime}$ and $p_{2}^{\prime}$ outgoing momenta with $p_{1}^{2} \equiv p_{1}^{\prime 2} \equiv m_{1}^{2}$ and $p_{2}^{2} \equiv p_{2}^{\prime 2} \equiv m_{2}^{2}$. We employ standard Mandelstam conventions throughout this presentation:

$s \equiv\left(p_{1}+p_{2}\right)^{2} \equiv\left(p_{1}^{\prime}+p_{2}^{\prime}\right)^{2}=m_{1}^{2}+m_{2}^{2}+2 m_{1} m_{2} \sigma$,

$\sigma \equiv \frac{p_{1} \cdot p_{2}}{m_{1} m_{2}}$,

\footnotetext{
${ }^{1}$ We thank Enrico Herrmann, Julio Parra-Martinez, Michael Ruf, and Mao Zeng for clarifying comments on this.
}

$t \equiv\left(p_{1}-p_{1}^{\prime}\right)^{2} \equiv\left(p_{2}^{\prime}-p_{2}\right)^{2} \equiv q^{2}=-\vec{q}^{2}$,

and

$$
u \equiv\left(p_{1}-p_{2}^{\prime}\right)^{2} \equiv\left(p_{1}^{\prime}-p_{2}\right)^{2}
$$

where $s$ defines the center of mass energy, $E_{\mathrm{CM}}^{2}$, and $t$ is the transfer momentum. One sees from the definitions that 


$$
\begin{aligned}
& p_{1} \cdot q=\frac{q^{2}}{2}, \quad p_{2} \cdot q=-\frac{q^{2}}{2}, \\
& p_{1}^{\prime} \cdot q=-\frac{q^{2}}{2}, \quad p_{2}^{\prime} \cdot q=\frac{q^{2}}{2} .
\end{aligned}
$$

In this section we recompute the classical contributions to the one-loop amplitude in Einstein gravity. This has already been done in many previous works but the method used here will be a good propaedeutic for the two-loop analysis that will be done in later in the paper.

We can expand the one-loop amplitude in Einstein gravity in terms of basis integrals

$$
\begin{aligned}
& \mathcal{M}^{1 \text {-loop }}\left(p_{1}, p_{2}, p_{1}^{\prime}, p_{2}^{\prime}\right) \\
& =i 64 \pi^{2} G_{N}^{2}\left(c_{\square} \mathcal{I}_{\square}+c_{\triangleright} \mathcal{I}_{\bowtie}+c_{\triangleright} \mathcal{I}_{\triangleright}+c_{\triangleleft} \mathcal{I}_{\triangleleft}+\cdots\right),
\end{aligned}
$$

spanned by the scalar box $I_{\square}$, the scalar cross box $I_{\bowtie}$, and scalar triangles $I_{\triangleleft}$ integral functions. The remainder in the amplitude are bubble integral and rational functions which do not contribute to the classical limit and give quantum mechanical contributions that we neglect. At leading order in the momentum transfer $\vec{q}$ the coefficients in $D$ dimensions are given by [22] (after reducing the quadratic triangle on scalar triangles)

$$
\begin{aligned}
& c_{\square}=c_{\bowtie}=16 m_{1}^{4} m_{2}^{4} \frac{\left(1-(D-2) \sigma^{2}\right)^{2}}{(D-2)^{2}}, \\
& c_{\triangleright}=\frac{4 m_{1}^{4} m_{2}^{2}\left((D-7)+(D(4 D-17)+19) \sigma^{2}\right)}{(D-2)^{2}}, \\
& c_{\triangleleft}=\frac{4 m_{1}^{2} m_{2}^{4}\left((D-7)+(D(4 D-17)+19) \sigma^{2}\right)}{(D-2)^{2}} .
\end{aligned}
$$

We consider the classical limit of the box and triangle integrals.

\section{A. Scalar triangle integrals}

We consider the classical limit of the scalar triangle integral

$$
\mathcal{I}_{\triangleright}\left(q^{2}\right)=\int \frac{d^{D} \ell}{(2 \pi \hbar)^{D}} \frac{\hbar^{4}}{\left((\ell+q)^{2}+i \varepsilon\right)\left(\ell^{2}+i \varepsilon\right)\left(\left(\ell+p_{1}\right)^{2}-m_{1}^{2}+i \varepsilon\right)},
$$

we symmetrize this expression over $p_{1}$ and $p_{1}^{\prime}$

$$
\mathcal{I}_{\triangleright}\left(q^{2}\right)=\frac{1}{2 \hbar^{D-4}} \int \frac{d^{D} \ell}{(2 \pi)^{D}} \frac{1}{\left((\ell+q)^{2}+i \varepsilon\right)\left(\ell^{2}+i \varepsilon\right)}\left(\frac{1}{\left(\ell+p_{1}\right)^{2}-m_{1}^{2}+i \varepsilon}+\frac{1}{\left(\ell-p_{1}^{\prime}\right)^{2}-m_{1}^{2}+i \varepsilon}\right) .
$$

Using that $p_{1}^{2}=p_{1}^{\prime 2}=m_{1}^{2}$ and the notation $q=|\vec{q}| u_{q}$ so that $u_{q}^{2}=-1$, and rescaling the loop momentum $\ell=|\vec{q}| l$, and $\vec{q}=\hbar \vec{q}$, we have at leading order in $q^{2}$

$$
\mathcal{I}_{\triangleright}\left(q^{2}\right)=\frac{|q|^{D-5}}{2 \hbar} \int \frac{d^{D} l}{(2 \pi)^{D}} \frac{1}{\left(\left(l+u_{q}\right)^{2}+i \varepsilon\right)\left(l^{2}+i \varepsilon\right)}\left(\frac{1}{2 l \cdot p_{1}+i \varepsilon}-\frac{1}{2 l \cdot p_{1}-i \varepsilon}\right) .
$$

In this expression we have approximated the propagator

$$
\frac{1}{\left(\ell+p_{1}\right)^{2}-m_{1}^{2}+i \varepsilon}=\frac{1}{\ell^{2}+2 \ell \cdot p_{1}+i \varepsilon}=\frac{1}{\hbar^{2} l^{2}|\underline{q}|^{2}+2 \hbar|\underline{q}| l \cdot p_{1}+i \varepsilon} \simeq \frac{1}{2 \hbar|\underline{q}| l \cdot p_{1}+i \varepsilon},
$$

since the classical contribution will come from the leading order in $\hbar$ of this expression. Using the definition of the delta function as a distribution ${ }^{2}$

$$
\lim _{\varepsilon \rightarrow 0^{+}}\left(\frac{1}{x-i \varepsilon}-\frac{1}{x+i \varepsilon}\right)=\lim _{\varepsilon \rightarrow 0^{+}} \frac{2 i \varepsilon}{x^{2}+\varepsilon^{2}}=2 i \pi \delta(x) .
$$

We then have in the limit $\varepsilon \rightarrow 0$ that

\footnotetext{
${ }^{2}$ Our prescription for the propagators is $\frac{i}{p^{2}-m^{2}+i \varepsilon}=P P\left(\frac{i}{p^{2}-m^{2}}\right)-i \pi \delta\left(p^{2}-m^{2}\right)$ with $\varepsilon>0$ and the mostly minus signature $(+-\cdots-)$. This, together with the appropriate contour of integrations (cf. page 31 of [23]), is the way to regulate the pole in the Green function.
} 


$$
\mathcal{I}_{\triangleright}\left(q^{2}\right)=-\frac{|q|^{D-5}}{2 \hbar} \int \frac{d^{D} l}{(2 \pi)^{D}} \frac{2 i \pi \delta\left(2 l \cdot p_{1}\right)}{\left(\left(l+u_{q}\right)^{2}+i \varepsilon\right)\left(l^{2}+i \varepsilon\right)}+\mathcal{O}(\varepsilon) .
$$

The integral reduces to the scalar bubble integral in $D-1$ dimensions

$$
\mathcal{I}_{\triangleright}\left(q^{2}\right)=-\frac{i|q|^{D-5}}{4 m_{1} \hbar} \int \frac{d^{D-1} l}{(2 \pi)^{D-1}} \frac{1}{\left(l^{2}+i \varepsilon\right)\left(\left(l+u_{q}\right)^{2}+i \varepsilon\right)}+\mathcal{O}(\varepsilon),
$$

which can be easily computed to give

$$
\mathcal{I}_{\triangleright}\left(q^{2}\right)=\frac{|q|^{D-5}}{4 m_{1} \hbar} \frac{\Gamma\left(\frac{D-3}{2}\right) \Gamma\left(\frac{5-D}{2}\right)}{(4 \pi)^{\frac{D-1}{2}} \Gamma(D-3)} .
$$

In $D=4-2 \epsilon$ we get

$$
I_{\triangleright}\left(q^{2}\right)=\frac{1}{32 m_{1} \hbar|\underline{q}|}+\mathcal{O}(\epsilon)
$$

\section{B. Box integral}

The scalar box integral is defined as

$$
\mathcal{I}_{\square}=\int \frac{d^{D} \ell}{(2 \pi \hbar)^{D}} \frac{\hbar^{4}}{\left(\left(\ell+p_{1}\right)^{2}-m_{1}^{2}+i \varepsilon\right)\left(\left(\ell-p_{2}\right)^{2}-m_{2}^{2}+i \varepsilon\right)\left(\ell^{2}+i \varepsilon\right)\left((\ell+q)^{2}+i \varepsilon\right)},
$$

and the scalar cross box is

$$
\mathcal{I}_{\bowtie}=\int \frac{d^{D} \ell}{(2 \pi \hbar)^{D}} \frac{\hbar^{4}}{\left(\left(\ell+p_{1}\right)^{2}-m_{1}^{2}+i \varepsilon\right)\left(\left(\ell+p_{2}^{\prime}\right)^{2}-m_{2}^{2}+i \varepsilon\right)\left(\ell^{2}+i \varepsilon\right)\left((\ell+q)^{2}+i \varepsilon\right)} .
$$

After symmetrization on the external legs the sum of the box and the cross-box contributions $\mathcal{I}_{\square}=\mathcal{I}_{\square}+\mathcal{I}_{\bowtie}$ reads

$$
\begin{aligned}
\mathcal{I}_{\square}= & \int \frac{d^{D} \ell}{(2 \pi \hbar)^{D}} \frac{\hbar^{4}}{\left(\ell^{2}+i \varepsilon\right)\left((\ell+q)^{2}+i \varepsilon\right)}\left(\frac{1}{\left(\ell+p_{1}\right)^{2}-m_{1}^{2}+i \varepsilon}+\frac{1}{\left(\ell-p_{1}^{\prime}\right)^{2}-m_{1}^{2}+i \varepsilon}\right) \\
& \times\left(\frac{1}{\left(\ell-p_{2}\right)^{2}-m_{2}^{2}+i \varepsilon}+\frac{1}{\left(\ell+p_{2}^{\prime}\right)^{2}-m_{2}^{2}+i \varepsilon}\right) .
\end{aligned}
$$

As before we change variables $\ell \rightarrow|\vec{q}| l$ with $\vec{q}=\hbar \underline{\vec{q}}$ to get

$$
\begin{aligned}
\mathcal{I}_{\square}= & \frac{|\overrightarrow{\vec{q}}|^{D-6}}{\hbar^{2}} \int \frac{d^{D} l}{(2 \pi)^{D}} \frac{1}{\left(l^{2}+i \varepsilon\right)\left(\left(l+u_{q}\right)^{2}+i \varepsilon\right)}\left(\frac{1}{2 l \cdot p_{1}+\hbar l^{2}|\underline{\vec{q}}|+i \varepsilon}+\frac{1}{-2 l \cdot p_{1}+\hbar|\underline{\vec{q}}|\left(l+u_{q}\right)^{2}+i \varepsilon}\right) \\
& \times\left(\frac{1}{-2 l \cdot p_{2}+\hbar l^{2}|\underline{\vec{q}}|+i \varepsilon}+\frac{1}{2 l \cdot p_{2}+\hbar|\underline{\vec{q}}|\left(l+u_{q}\right)^{2}+i \varepsilon}\right),
\end{aligned}
$$

where we used that $p_{1}^{\prime}=p_{1}-q$ and $p_{2}^{\prime}=p_{2}+q$. As the box contribution is of order $1 / \hbar^{2}$ one needs to do the small $q=\hbar \underline{q}$ expansion of the integral of the second order

$$
\mathcal{I}_{\bigotimes}=\frac{|\overrightarrow{\vec{q}}|^{D-6}}{\hbar^{2}}\left(\mathcal{I}_{\square}^{0}+\hbar|\underline{\vec{q}}| \mathcal{I}_{\bigotimes}^{1}+(\hbar|\underline{\vec{q}}|)^{2} \mathcal{I}_{\square}^{2}+O\left((\hbar \underline{q})^{3}\right)\right) .
$$

For doing this expansion we change variables to $p_{1}=\bar{p}_{1}+\frac{\hbar q}{2}$ and $p_{2}=\bar{p}_{2}-\frac{\hbar q}{2}$. 


\section{The leading contribution}

The leading contribution is given by

$\mathcal{I}_{\square}^{0}=-\frac{1}{2} \int \frac{d^{D} l}{(2 \pi)^{D-2}} \frac{\delta\left(2 \bar{p}_{1} \cdot l\right) \delta\left(2 \bar{p}_{2} \cdot l\right)}{\left(l^{2}+i \varepsilon\right)\left(\left(l+u_{q}\right)^{2}+i \varepsilon\right)}+\mathcal{O}(\varepsilon)$,

where we made use of the result in Eq. (18). The deltafunction constraints imply to divide by the determinant of the two vectors $\left(\epsilon_{\mu_{1} \mu_{2}} \bar{p}_{1}^{\mu_{1}} \bar{p}_{2}^{\mu_{2}}\right)^{2}=\left(\bar{p}_{1} \cdot \bar{p}_{2}\right)^{2}-\bar{p}_{1}^{2} \bar{p}_{2}^{2}=$ $m_{1}^{2} m_{2}^{2}\left(\sigma^{2}-1-\frac{(\hbar|q|)^{2} s}{4 m_{1}^{2} m_{2}^{2}}\right)$, to give

$$
\begin{aligned}
\mathcal{I}_{\square}^{0}= & -\frac{1}{8 m_{1} m_{2} \sqrt{\sigma^{2}-1-\frac{(\hbar|q|)^{2} s}{4 m_{1}^{2} m_{2}^{2}}}} \\
& \times \int \frac{d^{D-2} l}{(2 \pi)^{D-2}} \frac{1}{\left(l^{2}+i \varepsilon\right)\left(\left(l+u_{q}\right)^{2}+i \varepsilon\right)}+\mathcal{O}(\varepsilon),
\end{aligned}
$$

using the result given in Appendix A of [24]

$$
\int \frac{d^{D-2} l}{l^{2}\left(l+u_{q}\right)^{2}}=\frac{\pi^{\frac{D-2}{2}} \Gamma\left(\frac{D-4}{2}\right)^{2} \Gamma\left(\frac{6-D}{2}\right)}{\Gamma(D-4)},
$$

we have for the leading order

$$
\mathcal{I}_{\square}^{0}=-\frac{1}{8 m_{1} m_{2} \sqrt{\sigma^{2}-1-\frac{(\hbar|q|)^{2} s}{4 m_{1}^{2} m_{2}^{2}}}} \frac{\pi^{\frac{D-2}{2}} \Gamma\left(\frac{D-4}{2}\right)^{2} \Gamma\left(\frac{6-D}{2}\right)}{(2 \pi)^{D-2} \Gamma(D-4)} .
$$

\section{The next-to-leading contribution}

The next-to-leading contribution in the expansion is obtained after applying Eq. (18) and reads

$$
\begin{aligned}
\mathcal{I}_{\square}^{1}= & \frac{i}{2} \int \frac{d^{D} l}{(2 \pi)^{D-1}} \frac{1}{\left(l^{2}+i \varepsilon\right)\left(\left(l+u_{q}\right)^{2}+i \varepsilon\right)} \\
& \times\left(\delta\left(2 \bar{p}_{2} \cdot l\right)\left(\frac{u_{q} \cdot l}{\left(2 \bar{p}_{1} \cdot l-i \varepsilon\right)^{2}}+\frac{u_{q} \cdot l}{\left(2 \bar{p}_{1} \cdot l+i \varepsilon\right)^{2}}\right)\right. \\
& \left.+\delta\left(2 \bar{p}_{1} \cdot l\right)\left(\frac{u_{q} \cdot l}{\left(2 \bar{p}_{2} \cdot l+i \varepsilon\right)^{2}}+\frac{u_{q} \cdot l}{\left(2 \bar{p}_{2} \cdot l-i \varepsilon\right)^{2}}\right)\right) \\
& +\mathcal{O}(\varepsilon),
\end{aligned}
$$

neglecting the tadpole contribution which does not contribute to the classical limit we get

$$
\begin{aligned}
\mathcal{I}_{\bigotimes}^{1} \simeq & \frac{i}{2} \int \frac{d^{D} l}{(2 \pi)^{D-1}} \frac{1}{\left(l^{2}+i \varepsilon\right)\left(\left(l+u_{q}\right)^{2}+i \varepsilon\right)} \\
& \times\left(\frac{\delta\left(2 \bar{p}_{2} \cdot l\right)}{\left(2 \bar{p}_{1} \cdot l-i \varepsilon\right)^{2}}+\frac{\delta\left(2 \bar{p}_{1} \cdot l\right)}{\left(2 \bar{p}_{2} \cdot l+i \varepsilon\right)^{2}}\right)+\mathcal{O}(\varepsilon) .
\end{aligned}
$$

In the first integral we choose a vector $v$, with $v^{2}=-1$, orthogonal to $\bar{p}_{2}$ in the plane generated by $\bar{p}_{1}$ and $\bar{p}_{2}$. Then we have $\bar{p}_{1}=\frac{m_{1} \sigma \bar{p}_{2}}{m_{2}}-m_{1} \sqrt{\sigma^{2}-1} v$, such that $\bar{p}_{1} \cdot l=\frac{m_{1} \sigma \bar{p}_{2} \cdot l}{m_{2}}-m_{1} \sqrt{\sigma^{2}-1} v \cdot l$. Therefore in the first integral we have

$$
\frac{\delta\left(2 \bar{p}_{2} \cdot l\right)}{\left(2 \bar{p}_{1} \cdot l-i \varepsilon\right)^{2}}=\frac{\delta\left(2 \bar{p}_{2} \cdot l\right)}{\left(-2 m_{1} \sqrt{\sigma^{2}-1} v \cdot l-i \varepsilon\right)^{2}} .
$$

Similarly, in the second integral we choose a vector $v$, with $v^{2}=-1$, orthogonal to $\bar{p}_{1}$ in the plane generated by $\bar{p}_{1}$ and $\bar{p}_{2}$. Then we have $\bar{p}_{2}=\frac{m_{2} \sigma \bar{p}_{1}}{m_{1}}-m_{2} \sqrt{\sigma^{2}-1} v$ and $\bar{p}_{2} \cdot l=$ $\frac{m_{2} \sigma \bar{p}_{1} \cdot l}{m_{1}}-m_{2} \sqrt{\sigma^{2}-1} v \cdot l$ so that

$$
\frac{\delta\left(2 \bar{p}_{1} \cdot l\right)}{\left(2 \bar{p}_{2} \cdot l+i \varepsilon\right)^{2}}=\frac{\delta\left(2 \bar{p}_{1} \cdot l\right)}{\left(-2 m_{2} \sqrt{\sigma^{2}-1} v \cdot l+i \varepsilon\right)^{2}} .
$$

Taking into account the delta function, we have

$$
\begin{aligned}
\mathcal{I}_{\square}^{1}= & \frac{i\left(m_{1}+m_{2}\right)}{16 m_{1}^{2} m_{2}^{2}\left(\sigma^{2}-1\right)} \\
& \times \int \frac{d^{D-1} l}{(2 \pi)^{D-1}} \frac{1}{(v \cdot l)^{2}\left(l^{2}+i \varepsilon\right)\left(\left(l+u_{q}\right)^{2}+i \varepsilon\right)} \\
& +\mathcal{O}\left(\varepsilon, \underline{q}^{2}\right) .
\end{aligned}
$$

Using the result from Appendix A of [24]

$$
\begin{aligned}
\int \frac{d^{D-1} l}{(p \cdot l)^{2} l^{2}\left(l+u_{q}\right)^{2}} & =-\frac{2 \pi^{\frac{D-1}{2}} \Gamma\left(\frac{D-5}{2}\right)^{2} \Gamma\left(\frac{7-D}{2}\right)}{\Gamma(D-5)} \\
& =\frac{4 \pi^{\frac{D-1}{2}} \Gamma\left(\frac{D-3}{2}\right)^{2} \Gamma\left(\frac{5-D}{2}\right)}{\Gamma(D-4)},
\end{aligned}
$$

we get that

$$
\mathcal{I}_{\bigotimes}^{1}=\frac{i\left(m_{1}+m_{2}\right)}{16 m_{1}^{2} m_{2}^{2}\left(\sigma^{2}-1-\frac{(\hbar|q|)^{2} s}{4 m_{1}^{2} m_{2}^{2}}\right)} \frac{4 \pi^{\frac{D-1}{2}} \Gamma\left(\frac{D-3}{2}\right)^{2} \Gamma\left(\frac{5-D}{2}\right)}{(2 \pi)^{D-1} \Gamma(D-4)} .
$$

\section{The next-to-next-leading contribution}

The next-to-next-leading contribution is of order $(\hbar|q|)^{2}$. This is obtained by expanding the massive propagators 


$$
\begin{aligned}
\mathcal{I}_{\square}^{2}= & \frac{-1}{8 \hbar^{2}} \int \frac{d^{D} l}{(2 \pi)^{D}}\left(\frac{\frac{1}{4}\left(u_{q} \cdot l\right)^{2}}{\left(\overline{p_{1}} \cdot l-i \varepsilon\right)^{3}}-\frac{\frac{1}{4}\left(u_{q} \cdot l\right)^{2}}{\left(\overline{p_{1}} \cdot l+i \varepsilon\right)^{3}}\right)\left(\frac{1}{\overline{p_{2}} \cdot l+i \varepsilon}-\frac{1}{\overline{p_{2}} \cdot l-i \varepsilon}\right) \frac{1}{l^{2}\left(l+u_{q}\right)^{2}} \\
& \times \frac{-1}{8 \hbar^{2}} \int \frac{d^{D} l}{(2 \pi)^{D}}\left(\frac{1}{\overline{p_{1}} \cdot l-i \varepsilon}-\frac{1}{\overline{p_{1}} \cdot l+i \varepsilon}\right)\left(\frac{\frac{1}{4}\left(u_{q} \cdot l\right)^{2}}{\left(\overline{p_{2}} \cdot l+i \varepsilon\right)^{3}}-\frac{\frac{1}{4}\left(u_{q} \cdot l\right)^{2}}{\left(\overline{p_{2}} \cdot l-i \varepsilon\right)^{3}}\right) \frac{1}{l^{2}\left(l+u_{q}\right)^{2}} \\
& \times \frac{-1}{8 \hbar^{2}} \int \frac{d^{D} l}{(2 \pi)^{D}}\left(\frac{-\frac{1}{2} u_{q} \cdot l}{\left(\overline{p_{1}} \cdot l-i \varepsilon\right)^{2}}-\frac{\frac{1}{2} u_{q} \cdot l}{\left(\overline{p_{1}} \cdot l+i \varepsilon\right)^{2}}\right)\left(\frac{\frac{1}{2} u_{q} \cdot l}{\left(\overline{p_{2}} \cdot l+i \varepsilon\right)^{2}}+\frac{\frac{1}{2} u_{q} \cdot l}{\left(\overline{p_{2}} \cdot l-i \varepsilon\right)^{2}}\right) \frac{1}{l^{2}\left(l+u_{q}\right)^{2}} .
\end{aligned}
$$

Using the principal part identity Eq. (18) and LITERED [25] for reducing the integrals to one-loop master integrals, we obtain

$$
\mathcal{I}_{\square}^{2}=\frac{(4 \pi)^{\epsilon}(1+2 \epsilon) \Gamma(1+\epsilon) \Gamma(-\epsilon)^{2}}{64 \hbar^{2} \pi^{2} m_{1}^{2} m_{2}^{2}\left(\sigma^{2}-1\right)^{\frac{3}{2}} \Gamma(-2 \epsilon)}\left(\frac{s \pi}{4 m_{1} m_{2}}+i\left(\sigma \operatorname{arccosh}(\sigma)-\sqrt{\sigma^{2}-1}\right)\right) .
$$

\section{Summary of the one-loop computation in Einstein gravity}

Summing these contributions one gets for the scalar box integral contributions in $D=4-2 \epsilon$

$$
\begin{aligned}
\mathcal{I}_{\bigotimes}= & \frac{1}{|\underline{q}|^{2+2 \epsilon}}\left(-\frac{(4 \pi)^{\epsilon} \Gamma(1+\epsilon) \Gamma(-\epsilon)^{2}}{32 \hbar^{2} \pi m_{1} m_{2} \Gamma(-2 \epsilon) \sqrt{\sigma^{2}-1-\frac{(\hbar|q|)^{2} s}{4 m_{1}^{2} m_{2}^{2}}}}+\frac{i(4 \pi)^{\epsilon}\left(m_{1}+m_{2}\right)|\vec{q}|}{32 \hbar m_{1}^{2} m_{2}^{2}\left(\sigma^{2}-1\right)} \frac{\Gamma\left(\frac{1}{2}-\epsilon\right)^{2} \Gamma\left(\frac{1}{2}+\epsilon\right)}{\pi^{\frac{3}{2}} \Gamma(-2 \epsilon)}\right. \\
& +\frac{(4 \pi)^{\epsilon}|\vec{q}|^{2} \Gamma(1+\epsilon) \Gamma(-\epsilon)^{2}}{64 \pi^{2} m_{1}^{2} m_{2}^{2}\left(\sigma^{2}-1\right)^{\frac{3}{2}} \Gamma(-2 \epsilon)}\left(\frac{s \pi(1+2 \epsilon)}{4 m_{1} m_{2}}+i(1+2 \epsilon)\left(\sigma \operatorname{arccosh}(\sigma)-\sqrt{\left.\sigma^{2}-1\right)}\right)+\mathcal{O}\left(|\vec{q}|^{3}\right)\right) .
\end{aligned}
$$

Expanding the denominator of the first term leads to ${ }^{3}$

$$
\begin{aligned}
\mathcal{I}_{\square}= & \frac{1}{|\underline{q}|^{2+2 \epsilon}}\left(-\frac{(4 \pi)^{\epsilon} \Gamma(1+\epsilon) \Gamma(-\epsilon)^{2}}{32 \hbar^{2} \pi m_{1} m_{2} \Gamma(-2 \epsilon) \sqrt{\sigma^{2}-1}}+\frac{i(4 \pi)^{\epsilon}\left(m_{1}+m_{2}\right)|\vec{q}|}{32 \hbar m_{1}^{2} m_{2}^{2}\left(\sigma^{2}-1\right)} \frac{\Gamma\left(\frac{1}{2}-\epsilon\right)^{2} \Gamma\left(\frac{1}{2}+\epsilon\right)}{\pi^{\frac{3}{2}} \Gamma(-2 \epsilon)}\right. \\
& \left.+\frac{(4 \pi)^{\epsilon}|\vec{q}|^{2} \Gamma(1+\epsilon) \Gamma(-\epsilon)^{2}}{64 \pi^{2} m_{1}^{2} m_{2}^{2}\left(\sigma^{2}-1\right)^{\frac{3}{2}} \Gamma(-2 \epsilon)}\left(\frac{\epsilon S \pi}{2 m_{1} m_{2}}+i(1+2 \epsilon)\left(\sigma \operatorname{arccosh}(\sigma)-\sqrt{\sigma^{2}-1}\right)\right)+\mathcal{O}\left(|\vec{q}|^{3}\right)\right) .
\end{aligned}
$$

This expression matches the sum of the expressions given in Eqs. (B.36) and (B.40) in [27] and Eqs. (4.54) and (4.59) in [12] up to a normalization constant. The result is valid in arbitrary dimension, and it keeps covariant vectors everywhere.

\section{Comparing the soft and the potential region}

We can now compare the result for the box to the one derived from the potential region and see why this leads to a different answer. In order to do the potential region versus the soft region analysis, we will define

$$
\Omega=\frac{p_{1}+p_{2}}{\sqrt{s}},
$$

which is a timelike vector orthogonal to $q$. We will also define the vector

\footnotetext{
${ }^{3}$ This expansion is justified as long as $\sigma \neq 1$, which is the hypothesis we will use since we are considering the scattering angle. In the static case $\sigma=1$ the amplitude reproduces the oneloop computation of [26].
}

$$
\tilde{v}_{\mu}=\frac{\epsilon_{\mu \nu \rho \sigma} p_{1}^{\nu} p_{2}^{\rho} u_{q}^{\sigma}}{m_{1} m_{2}}
$$

orthogonal to $p_{1}, p_{2}$, and $q$, and $v$ a unit vector $\left(v^{2}=-1\right)$ orthogonal to $\Omega, u_{q}$, and $\tilde{v}$. The vectors $\left(\Omega, u_{q}, v, \tilde{v}\right)$ form a basis of the four-dimensional Minkowski space, on which one can expand $p_{1}$ and $p_{2}$ as

$p_{1}=\frac{m_{1}^{2}+m_{1} m_{2} \sigma}{\sqrt{s}} \Omega+\frac{|\vec{q}|}{2} u_{q}+v \sqrt{\frac{m_{1}^{2} m_{2}^{2}\left(\sigma^{2}-1\right)}{s}-\frac{|\vec{q}|^{2}}{4}}$,

and

$p_{2}=\frac{m_{2}^{2}+m_{1} m_{2} \sigma}{\sqrt{s}} \Omega-\frac{|\vec{q}|}{2} u_{q}-v \sqrt{\frac{m_{1}^{2} m_{2}^{2}\left(\sigma^{2}-1\right)}{s}-\frac{|\vec{q}|^{2}}{4}}$.

The potential region is defined as the integration region where the time component of the loop momentum, 
$\omega \equiv \Omega \cdot l$ is very small compared to the spatial components. This will not have any consequences in the evaluation of the leading contribution $\mathcal{I}_{\square}^{0}$ in Eq. (31) because the delta functions in this integral always cancel the time component in the graviton propagators. But this will have effects on the subleading contribution $\mathcal{I}_{\square}^{1}$ in (32). In the potential region this contribution can be written at leading order in $|\underline{q}|$ as

$$
\begin{aligned}
\mathcal{I}_{\unrhd}^{1 p}= & -\frac{i|q|^{D-6}}{4 \hbar} \int \frac{d^{D} l}{(2 \pi)^{D}} \frac{1}{\left(\vec{l}^{2}-i \varepsilon\right)\left(\left(\vec{l}+\overrightarrow{u_{q}}\right)^{2}-i \varepsilon\right)} \\
& \times\left(\frac{\delta\left(\frac{m_{2}^{2}+m_{1} m_{2} \sigma}{\sqrt{s}} \omega-\sqrt{\frac{m_{1}^{2} m_{2}^{2}\left(\sigma^{2}-1\right)}{s}} v \cdot l\right)}{\left(2 \frac{m_{1}^{2}+m_{1} m_{2} \sigma}{\sqrt{s}} \omega+2 \sqrt{\frac{m_{1}^{2} m_{2}^{2}\left(\sigma^{2}-1\right)}{s}} v \cdot l-i \varepsilon\right)^{2}}+\frac{\delta\left(\frac{m_{1}^{2}+m_{1} m_{2} \sigma}{\sqrt{s}} \omega+\sqrt{\frac{m_{1}^{2} m_{2}^{2}\left(\sigma^{2}-1\right)}{s}} v \cdot l\right)}{\left(2 \frac{m_{2}^{2}+m_{1} m_{2} \sigma}{\sqrt{s}} \omega-2 \sqrt{\frac{m_{1}^{2} m_{2}^{2}\left(\sigma^{2}-1\right)}{s}} v \cdot l+i \varepsilon\right)^{2}}\right) .
\end{aligned}
$$

Integrating over the $\omega$ variable gives

$$
\mathcal{I}_{\square}^{1 p}=-\frac{i|q|^{D-6}}{4 \hbar} \int \frac{d^{D-1} l}{(2 \pi)^{D-1}} \frac{1}{\left(\vec{l}^{2}-i \varepsilon\right)\left(\left(\vec{l}+\overrightarrow{u_{q}}\right)^{2}-i \varepsilon\right)}\left(\frac{\frac{\sqrt{s}}{m_{2}^{2}+m_{1} m_{2} \sigma}}{\left(2 \frac{\sqrt{s m_{1}^{2} m_{2}^{2}\left(\sigma^{2}-1\right)}}{m_{2}^{2}+m_{1} m_{2} \sigma} v \cdot l-i \varepsilon\right)^{2}}+\frac{\frac{\sqrt{s}}{m_{2}^{2}+m_{1} m_{2} \sigma}}{\left(2 \frac{\sqrt{s m_{2}^{2} m_{2}^{2}\left(\sigma^{2}-1\right)}}{m_{1}^{2}+m_{1} m_{2} \sigma} \cdot l+i \varepsilon\right)^{2}}\right),
$$

leading to

$$
\mathcal{I}_{\square}^{1 p}=\frac{i|\underline{q}|^{D-6} \sqrt{s}}{16 \hbar m_{1}^{2} m_{2}^{2}\left(\sigma^{2}-1\right)} \int \frac{d^{D-1} l}{(2 \pi)^{D-1}} \frac{1}{(v \cdot l)^{2}\left(\vec{l}^{2}-i \varepsilon\right)\left(\left(\vec{l}+\vec{u}_{q}\right)^{2}-i \varepsilon\right)} .
$$

Since $\sigma=\frac{p_{1} \cdot p_{2}}{m_{1} m_{2}}$ we find that this integral is related to the box contribution in Eq. (38) as

$$
\mathcal{I}_{\square}^{1 p}=\frac{\sqrt{s}}{m_{1}+m_{2}} \mathcal{I}_{\square}^{1}
$$

This is exactly the difference noticed in Eq. (B.57) [27] between the evaluation in the potential region and the soft region at one-loop.

Actually the difference between the potential region $\mathcal{I}_{\square}^{1 p}$ is and the full (soft) integral $\mathcal{I}_{\unrhd}^{1}$ is the expansion of the internal graviton propagator with respect to the $\omega$

$$
\begin{aligned}
\mathcal{I}_{\square}^{1}-\mathcal{I}_{\square}^{1 p}= & \frac{i}{4 \hbar} \int \frac{d^{D} l}{(2 \pi)^{D-1}}\left(\frac{\delta\left(p_{2} \cdot l\right)}{\left(2 p_{1} \cdot l-i \varepsilon\right)^{2}}+\frac{\delta\left(p_{1} \cdot l\right)}{\left(2 p_{2} \cdot l+i \varepsilon\right)^{2}}\right) \\
& \times \sum_{n=1}^{\infty} \sum_{l=0}^{n} \frac{\omega^{2 n}}{\left(\vec{l}^{2}-i \varepsilon\right)^{(l+1)}\left(\left(\vec{l}+\overrightarrow{u_{q}}\right)^{2}-i \varepsilon\right)^{(n-l+1)}} .
\end{aligned}
$$

Although the exchange of the velocity expansion and integration does not lead to a different result at one-loop order, this will be different at the two-loop order. This will be apparent when comparing the potential region result with the amplitude computation done in this work.

\section{TWO-BODY AMPLITUDES IN MAXIMAL SUPERGRAVITY}

In this section, we summarize the evaluation of fourpoint maximal supergravity amplitudes with massive external states up to the third order in Newton's constant.

The generic maximal supergravity four-point amplitude takes the form of a helicity dependent kinematical factor $\mathbf{R}_{\zeta_{1}, \zeta_{2}, \zeta_{3}, \zeta_{4}}^{4}$ times a sum of loop integrals that does not depend on the helicities $\zeta_{i}$ of the external states

$$
\begin{aligned}
\mathcal{M}^{N=8} & \left(\zeta_{1}, \ldots, \zeta_{4}\right) \\
= & \mathbf{R}_{\zeta_{1}, \zeta_{2}, \zeta_{3}, \zeta_{4}}^{4}\left[\mathcal{M}_{0}\left(p_{1}, \ldots, p_{4}\right)+\mathcal{M}_{1}\left(p_{1}, \ldots, p_{4}\right)+\cdots\right. \\
& \left.+\mathcal{M}_{L}\left(p_{1}, \ldots, p_{4}\right)+\mathcal{O}\left(G_{N}^{L+2}\right)\right] .
\end{aligned}
$$

Following Eq. (7.4.57) of [28] we define

$$
\mathbf{R}_{\zeta_{1}, \zeta_{2}, \zeta_{3}, \zeta_{4}}^{4} \equiv \zeta_{1}^{A A^{\prime}} \zeta_{2}^{B B^{\prime}} \zeta_{3}^{C C^{\prime}} \zeta_{4}^{D D^{\prime}} K_{A B C D} \tilde{K}_{A^{\prime} B^{\prime} C^{\prime} D^{\prime}},
$$

where the indices $A, B$ on the polarization tensor $\zeta_{r}^{A B}$ run over vector and spinor values. The tensors $K$ and $\tilde{K}$ are expressed as traces of the fermionic zero modes as shown in Appendix 9.A of [29]. For external graviton states the tensors $K$ and $\tilde{K}$ are given by the expression in Eq. (9.A.18) of [29] and one obtains the linearization of the $R^{4}$-kinematic factor and $\mathbf{R}_{\zeta_{1}, \zeta_{2}, \zeta_{3}, \zeta_{4}}^{4}=\prod_{r=1}^{4} \hat{R}^{(r) \mu_{2 r-1} \mu_{2 r} \nu_{2 r-1} \nu_{2 r}} t_{\mu_{1} \cdots \mu_{8}}^{8} t_{\nu_{1} \cdots \nu_{8}}^{8}$ where $\hat{R}_{\mu \nu \rho \sigma}=-4 p_{[\mu} \zeta_{\nu][\rho} p_{\sigma]}$ is the linearized Riemann 
tensor. We obtain external massive states by considering Kaluza-Klein reduction [30]. The kinematic variable then becomes

$$
\begin{aligned}
& S=s-m_{1}^{2}-m_{2}^{2}=2 m_{1} m_{2} \sigma, \quad T=t=\hbar^{2} \underline{q}^{2}, \\
& U=u-m_{1}^{2}-m_{2}^{2}=-2 m_{1} m_{2} \sigma-\hbar^{2} \underline{q}^{2} .
\end{aligned}
$$

Since this construction preserves maximal supersymmetry, the helicity dependence in Eq. (52) is preserved. For the choice of helicity states in $[11,15]$ the kinematical factor is

$$
\begin{aligned}
\mathbf{R}_{\phi, \phi, \varphi, \varphi}^{4}= & \frac{1}{2}\left(S^{4}+T^{4}+U^{4}\right) \\
= & 16 m_{1}^{4} m_{2}^{4} \sigma^{4}-16 m_{1}^{3} m_{2}^{3} \underline{q}^{2} \sigma^{3} \hbar^{2} \\
& +12 m_{1}^{2} m_{2}^{2}\left(\underline{q^{2}}\right)^{2} \sigma^{2} \hbar^{4}-4 m_{1} m_{2} \sigma\left(\underline{q^{2}}\right)^{3} \hbar^{6} \\
& +\left(\underline{q}^{2}\right)^{4} \hbar^{8},
\end{aligned}
$$

whereas for the choice of helicities in $[12,30]$ the kinematical factor is given by

$$
\mathbf{R}_{\phi, \phi, \phi, \phi}^{4}=S^{4}=16 m_{1}^{4} m_{2}^{4} \sigma^{4},
$$

where we used the kinematical relations in Eqs. (8)-(10) and the definition of the momentum transfer $\vec{q} \equiv \hbar \underline{\vec{q}}$ tailored for extracting classical physics from amplitudes.

Focussing on small momentum transfer $|\underline{q}| \ll m_{1}, m_{2}$ the $L$-order loop amplitude takes the form

$$
\mathcal{M}_{L}(\sigma,|\underline{q}|)=\frac{1}{\hbar^{L-1}|\underline{q}|^{2 L \epsilon}} \sum_{r \geq-2} \mathcal{M}_{L}^{(r)}(\sigma, \epsilon)(\hbar|\underline{q}|)^{r} .
$$

We justify the expansion by the following counting. The leading singular term in the classical limit $\hbar \rightarrow 0$ arises from the ladder and cross-ladder diagrams at $L$ loop. These diagram have the maximal number of massive propagators, with $2 L$ massive propagators and $L+1$ massless gravitons exchanges, so the loop integrals symbolically read (see Secs. III A and III for details about this at one-loop and two-loop order amplitude, respectively)

$\left.\mathcal{M}_{L}(\sigma,|q|)\right|_{\mathrm{ladder}} \sim \int \prod_{i=1}^{L} \frac{d^{D} \ell}{(2 \pi \hbar)^{D}} \frac{\hbar^{3 L+1}}{\left(2 \ell_{i} \cdot p_{j}\right)^{2 L}\left(\ell_{i}^{2}\right)^{L+1}}$,

and the classical limit is obtained for $\hbar \rightarrow 0$ keeping $q / \hbar=q$ constant. Rescaling the loop momenta by $\ell=$ $\hbar q l$ we get

$\left.\mathcal{M}_{L}(\sigma,|q|)\right|_{\text {ladder }} \sim \frac{1}{\hbar^{L-1}|\underline{q}|^{2 L \epsilon}} \frac{1}{(\hbar \underline{q})^{2}} \int \frac{\prod_{i=1}^{L} d^{D} l}{\left(2 l_{i} \cdot p_{j}\right)^{2 L}\left(l_{i}^{2}\right)^{L+1}}$.
At $L$-loop order, the other diagrams have more massless propagators and less massive propagators. Replacing a massive propagator by a massless propagator amounts to multiplying the $\hbar$ count by a factor of $\hbar q$, giving the expansion in Eq. (57). The least number of massive propagator at $L$-loop order is two, so with $3 L-1$ massless propagators we have the $\hbar$ counting

$$
\frac{1}{\hbar^{L-1}|\underline{q}|^{2 L \epsilon}} \frac{1}{(\hbar|\underline{q}|)^{3 L-1}} .
$$

We note that such a contribution will come from the $t$ channel with an extra power of the momentum in the numerator contributing to $(\hbar|q|)^{2 L}$. This will be the case for the $J_{t}$ contribution at two-loop in Eq. (117).

\section{A. The amplitudes in momentum space}

We give the expression of the tree-level amplitude in Sec. III A 1, one-loop amplitude in Sec. III A 2, and twoloop amplitude in Sec. III A 3.

\section{Tree-level scalar amplitude}

The helicity independent factor in the massless maximal supergravity four-point tree-level amplitude is given by

$$
\mathcal{M}_{0}\left(p_{1}, \ldots, p_{4}\right)=\frac{8 \pi G_{N} \hbar}{S T U},
$$

which after Kaluza-Klein reduction in Eq. (54) gives the two-body scattering amplitude

$$
\begin{aligned}
\mathcal{M}_{0}(\sigma,|\underline{q}|) & =-\frac{8 \pi G_{N}}{2 m_{1} m_{2} \hbar|\underline{q}|^{2} \sigma\left(\hbar^{2}|\underline{q}|^{2}-2 m_{1} m_{2} \sigma\right)} \\
& =\hbar \frac{\mathcal{M}_{0}^{(-2)}(\sigma)}{\hbar^{2}|\underline{q}|^{2}}+\mathcal{O}\left((\hbar|\underline{q}|)^{0}\right),
\end{aligned}
$$

where

$$
\mathcal{M}_{0}^{(-2)}(\sigma)=\frac{2 \pi G_{N}}{m_{1}^{2} m_{2}^{2} \sigma^{2}} .
$$

\section{One-loop scalar amplitudes}

The helicity independent part of the one-loop amplitude is the sum of the scalar box and cross-box integrals

$$
\mathcal{M}_{1}\left(p_{1}, p_{2}, p_{1}^{\prime}, p_{2}^{\prime}\right)=-i\left(8 \pi G_{N}\right)^{2}\left(\mathcal{I}_{\square}+\mathcal{I}_{\bowtie}\right) .
$$

[This result is dimensional reduction of the maximal supergravity massless one-loop amplitude in [31] without the $(T, U)$-channel contribution which vanishes because of choice of helicity configuration.]

The boxes have been evaluated in Sec. III A with the result in (42) 


$$
\mathcal{M}_{1}(\sigma,|\underline{q}|)=\frac{1}{|\underline{q}|^{2 \epsilon}}\left(\frac{\mathcal{M}_{1}^{(-2)}(\sigma, \epsilon)}{\hbar^{2}|\underline{q}|^{2}}+\frac{\mathcal{M}_{1}^{(-1)}(\sigma, \epsilon)}{\hbar|\underline{q}|}+\mathcal{M}_{1}^{(0)}(\sigma, \epsilon)+\mathcal{O}(\hbar|\underline{q}|)\right),
$$

with the coefficient to all orders in $\epsilon$

$$
\begin{gathered}
\mathcal{M}_{1}^{(-2)}(\sigma, \epsilon)=\frac{2 \pi i G_{N}^{2}}{m_{1} m_{2} \sqrt{\sigma^{2}-1}} \frac{(4 \pi)^{\epsilon} \Gamma(-\epsilon)^{2} \Gamma(\epsilon+1)}{\Gamma(-2 \epsilon)}, \\
\mathcal{M}_{1}^{(-1)}(\sigma, \epsilon)=\frac{2 \sqrt{\pi}\left(m_{1}+m_{2}\right) G_{N}^{2}}{m_{1}^{2} m_{2}^{2}\left(\sigma^{2}-1\right)} \frac{(4 \pi)^{\epsilon} \Gamma\left(\frac{1}{2}-\epsilon\right)^{2} \Gamma\left(\epsilon+\frac{1}{2}\right)}{\Gamma(-2 \epsilon)}, \\
\mathcal{M}_{1}^{(0)}(\sigma, \epsilon)=\frac{(4 \pi)^{\epsilon} \Gamma(-\epsilon)^{2} \Gamma(\epsilon+1)}{\Gamma(-2 \epsilon)} \frac{G_{N}^{2}\left(2 m_{1} m_{2}\left(\sigma \operatorname{arccosh}(\sigma)-\sqrt{\sigma^{2}-1}\right)(2 \epsilon+1)-i \pi s \epsilon\right)}{2 m_{1}^{3} m_{2}^{3}\left(\sigma^{2}-1\right)^{\frac{3}{2}}} .
\end{gathered}
$$

\section{Two-loop scalar amplitudes}

The helicity independent part of the two-loop amplitude four points amplitude is the Kaluza-Klein reduction of the maximal supergravity two-loop evaluated in [32], with the $(T, U)$ sector vanishing as being forbidden by the helicity configurations [12]

$$
\mathcal{M}_{2}(\sigma,|\underline{q}|)=\frac{1}{|\underline{\vec{q}}|^{4 \epsilon} \hbar}\left(\frac{\mathcal{M}_{2}^{(-2)}(\sigma, \epsilon)}{\hbar^{2}|\underline{q}|^{2}}+\frac{\mathcal{M}_{2}^{(-1)}(\sigma, \epsilon)}{\hbar|\underline{q}|}+M_{2}^{(0)}(\sigma, \epsilon)+\mathcal{O}(\hbar|\underline{q}|)\right),
$$

with $^{4}$

$$
\begin{gathered}
\mathcal{M}_{2}^{(-2)}(\sigma, \epsilon)=-\frac{G_{N}^{3} \sigma^{2}}{3\left(\sigma^{2}-1\right)} \frac{(4 \pi)^{1+2 \epsilon} \Gamma(-\epsilon)^{3} \Gamma(1+2 \epsilon)}{\Gamma(-3 \epsilon)}, \\
\mathcal{M}_{2}^{(-1)}(\sigma, \epsilon)=\frac{4 \sqrt{\pi} i G_{N}^{3}\left(m_{1}+m_{2}\right) \sigma^{2}}{m_{1} m_{2}\left(\sigma^{2}-1\right)^{\frac{3}{2}}} \frac{(4 \pi)^{2 \epsilon} \Gamma\left(\frac{1}{2}-\epsilon\right)^{2} \Gamma\left(\frac{1}{2}+2 \epsilon\right) \Gamma(-\epsilon) \Gamma\left(\frac{1}{2}-2 \epsilon\right)}{\Gamma\left(\frac{1}{2}-3 \epsilon\right) \Gamma(-2 \epsilon)},
\end{gathered}
$$

to all orders in $\epsilon$ and

$$
\begin{aligned}
\mathcal{M}_{2}^{(0)}(\sigma, \epsilon)= & \frac{2 G_{N}^{3}\left(4 \pi e^{-\gamma_{E}}\right)^{2 \epsilon}}{\pi m_{1} m_{2}\left(\sigma^{2}-1\right)^{2}}\left[\frac{i \pi(1+2 \epsilon) \sigma^{2}\left(\sigma \operatorname{arccosh}(\sigma)-\sqrt{\sigma^{2}-1}\right)}{\epsilon^{2}}+\frac{\pi^{2} s \sigma^{2}}{2 \epsilon m_{1} m_{2}}\right. \\
& -\frac{\pi^{2}\left(\sigma^{2}-1\right)^{\frac{3}{2}} \operatorname{arccosh}(\sigma)}{\epsilon}-\frac{i \pi}{\epsilon^{2}}\left(\frac{-1}{4\left(\sigma^{2}-1\right)}\right)^{\epsilon}\left((1+2 \epsilon) \sigma^{2} \sqrt{\sigma^{2}-1}+\sigma\left(\sigma^{2}-2\right) \operatorname{arccosh}(\sigma)\right. \\
& \left.\left.+\epsilon\left(\left(\sigma^{2}-1\right)^{\frac{3}{2}}-\sigma\left(\sigma^{2}-2\right)\right) \operatorname{arccosh}^{2}(\sigma)-\epsilon \sigma\left(\sigma^{2}-2\right) \operatorname{Li}_{2}\left(2-2 \sigma\left(\sigma+\sqrt{\sigma^{2}-1}\right)\right)\right)+\mathcal{O}(1)\right],
\end{aligned}
$$

where $(-1)^{\epsilon}=1+i \pi \epsilon+\mathcal{O}\left(\epsilon^{2}\right)$ and $\mathcal{O}(1)$ is defined so that it is regular function both at $\epsilon=0$ and $\sigma=1$. The details of this evaluation are given in Sec. IV.

\footnotetext{
${ }^{4}$ The expression for $\mathcal{M}_{2}^{(-1)}(\sigma, \epsilon)$ differs by a sign with the one given in Eq. (5.8) of [12]. The present sign guaranties the exponentiation of the two-loop amplitude.
} 


\section{B. The amplitudes in $b$ space}

The amplitude is $b$ space is defined by

$$
\begin{aligned}
\widetilde{\mathcal{M}_{L}^{N=}=8}(\sigma, b)= & \frac{1}{4 E_{\text {c.m. }} P} \int_{\mathbb{R}^{D-2}} \frac{d^{D-2} \underline{\vec{q}}}{(2 \pi)^{D-2}} \\
& \times \mathbf{R}_{\zeta_{1}, \ldots, \zeta_{4}}^{4} \mathcal{M}_{L}^{N=8}\left(p_{1}, p_{2}, p_{1}^{\prime}, p_{2}^{\prime}\right) e^{i \vec{q} \cdot \vec{b}},
\end{aligned}
$$

where $4 E_{\text {c.m. }} P=4 m_{1} m_{2} \sqrt{\sigma^{2}-1}$.

For the choice of helicity in $[12,30]$ the kinematic factor is independent of the momentum transfer $\vec{q}$ and therefore

$$
\begin{aligned}
\widetilde{\mathcal{M}_{L}^{N}} & =8 \\
& =\frac{4 m_{1}^{3} m_{2}^{3} \sigma^{4}}{\sqrt{\sigma^{2}-1}} \int \frac{d^{D-2} \underline{\underline{q}}}{(2 \pi)^{D-2}} \mathcal{M}_{L}\left(p_{1}, p_{2}, p_{1}^{\prime}, p_{2}^{\prime}\right) e^{i \vec{q} \cdot \vec{b}} .
\end{aligned}
$$

Using the Fourier transformation

$$
\int \frac{d^{D-2} \overrightarrow{\vec{q}}}{(2 \pi)^{D-2}} \frac{e^{i \vec{q} \cdot \vec{b}}}{|\underline{\vec{q}}|^{2 \alpha}}=\frac{\Gamma(1-\alpha-\epsilon)}{(4 \pi)^{1-\epsilon} \Gamma(\alpha)}\left(\frac{b}{2}\right)^{2 \alpha-2+2 \epsilon},
$$

the expansion in Eq. (57) reads

$$
\begin{aligned}
\widetilde{\mathcal{M}_{L}^{N}=8} & (\phi, \phi, \phi, \phi) \\
= & \frac{1}{\pi \hbar^{L-1} b^{2}}\left(\frac{b^{2(L+1)} \pi}{4^{L}}\right)^{\epsilon} \frac{4 m_{1}^{3} m_{2}^{3} \sigma^{4}}{\sqrt{\sigma^{2}-1}} \sum_{r \geq-2} \mathcal{M}_{L}^{(r)}(\sigma, \epsilon)\left(\frac{2 \hbar}{b}\right)^{r} \\
& \times \frac{\Gamma\left(\frac{r}{2}-L \epsilon-\epsilon+1\right)}{\Gamma\left(L \epsilon-\frac{r}{2}\right)} .
\end{aligned}
$$

For the choice of helicity in $[11,15]$ with the kinematic factor in Eq. (55) we have

$$
\begin{aligned}
\widetilde{\mathcal{M}_{L}^{N}=8} & (\phi, \phi, \varphi, \varphi) \\
= & \frac{4 m_{1}^{3} m_{2}^{3} \sigma^{4}}{\sqrt{\sigma^{2}-1}} \int \frac{d^{D-2} \overrightarrow{\vec{q}}}{(2 \pi)^{D-2}}\left(1-\frac{\hbar^{2} \underline{q}^{2}}{m_{1} m_{2} \sigma}+\mathcal{O}(\hbar \underline{q})^{3}\right) \\
& \times \mathcal{M}_{L}\left(p_{1}, p_{2}, p_{1}^{\prime}, p_{2}^{\prime}\right) e^{i \vec{i} \cdot \vec{b}} .
\end{aligned}
$$

We will show that the choice of helicity does not affect the classical piece of the amplitude.

\section{Tree-level amplitude}

Using the expansion given previously we have for the tree amplitude

$\widetilde{\mathcal{M}_{0}^{N=}=8}(\phi, \phi, \phi, \phi)=\frac{1}{\hbar} \frac{2 m_{1} m_{2} \sigma^{2} G_{N}}{\sqrt{\sigma^{2}-1}}(b \sqrt{\pi})^{2 \epsilon} \Gamma(-\epsilon)$.

We notice that this expression is actually exact because any higher order terms in the $|q|^{2}$ expansion in the tree-level amplitude has a vanishing Fourier transform.

As a consequence of the vanishing of any higher power correction in $|q|^{2}$, we have for the helicity choice in $[11,15]$ with the kinematic factor in Eq. (55)

$$
\widetilde{\mathcal{M}_{0}^{N=8}}(\phi, \phi, \varphi, \varphi)=\widetilde{\mathcal{M}_{0}^{N}=8}(\phi, \phi, \phi, \phi) .
$$

\section{One-loop amplitude}

For the one-loop amplitude to all order in $\epsilon$

$$
\begin{aligned}
\widetilde{\mathcal{M}_{1}^{N=}=8}(\phi, \phi, \phi, \phi)= & \left.\frac{i}{2} \widetilde{\mathcal{M}_{0}^{N=}=8}(\phi, \phi, \phi, \phi)\right)^{2} \\
& +\left.\widetilde{\mathcal{M}_{1}^{N}=8}(\phi, \phi, \phi, \phi)\right|_{\mathrm{Cl}} . \\
& +\left.\widetilde{\mathcal{M}_{1}^{N}=8}(\phi, \phi, \phi, \phi)\right|_{\mathrm{Q} t} .
\end{aligned}
$$

Where the one-loop classical part is given by

$$
\begin{aligned}
\left.\widetilde{\mathcal{M}_{1}^{N=8}}(\phi, \phi, \phi, \phi)\right|_{\mathrm{Cl} .} & =\frac{1}{4 \pi}\left(\frac{b^{2} \sqrt{\pi}}{2}\right)^{2 \epsilon} \frac{4 m_{1}^{3} m_{2}^{3} \sigma^{4}}{\sqrt{\sigma^{2}-1}} \frac{2 \mathcal{M}_{1}^{(-1)}(\sigma, \epsilon)}{\hbar b} \frac{\Gamma\left(\frac{1}{2}-2 \epsilon\right)}{\Gamma\left(\frac{1}{2}+\epsilon\right)} \\
& =\frac{4 G_{N}^{2} m_{1} m_{2} \sigma^{4}\left(m_{1}+m_{2}\right)}{\left(\sigma^{2}-1\right)^{\frac{3}{2}} \hbar} \frac{\left(b^{2} \pi\right)^{2 \epsilon-\frac{1}{2}} \Gamma\left(\frac{1}{2}-2 \epsilon\right) \Gamma\left(\frac{1}{2}-\epsilon\right)^{2}}{\Gamma(-2 \epsilon)}
\end{aligned}
$$

and the quantum piece of the one-loop amplitude is given by

$$
\begin{aligned}
\left.\widetilde{\mathcal{M}_{1}^{N=8}}(\phi, \phi, \phi, \phi)\right|_{\mathrm{Qt.}}= & \frac{1}{4 \pi}\left(\frac{b^{2} \sqrt{\pi}}{2}\right)^{2 \epsilon} \frac{4 m_{1}^{3} m_{2}^{3} \sigma^{4}}{\sqrt{\sigma^{2}-1}} \frac{4 \mathcal{M}_{1}^{(0)}(\sigma, \epsilon)}{b^{2}} \frac{\Gamma(1-2 \epsilon)}{\Gamma(\epsilon)} \\
= & -\frac{4 i G_{N}^{2} m_{1} m_{2} \sigma^{4}\left(\frac{\pi s \epsilon}{2 m_{1} m_{2}}+i(2 \epsilon+1)\left(\sigma \operatorname{arccosh}(\sigma)-\sqrt{\sigma^{2}-1}\right)\right)}{\left(\sigma^{2}-1\right)^{2}} \\
& \times \frac{\left(b^{2} \pi\right)^{2 \epsilon-1} \Gamma(1-2 \epsilon) \Gamma(-\epsilon)^{2} \Gamma(\epsilon+1)}{\Gamma(-2 \epsilon) \Gamma(\epsilon)} .
\end{aligned}
$$


For the choice of helicity in $[11,15]$ with the kinematic factor in Eq. (55)

$$
\left.\widetilde{\mathcal{M}_{1}^{N=8}}(\phi, \phi, \varphi, \varphi)\right|_{\mathrm{Cl} .}=\left.\widetilde{\mathcal{M}_{1}^{N=8}}(\phi, \phi, \phi, \phi)\right|_{\mathrm{Cl} .}
$$

$$
\begin{aligned}
& \widetilde{\mathcal{M}_{1}^{N}=8}(\phi, \phi, \varphi, \varphi) \\
& \quad=\widetilde{\mathcal{M}_{1}^{N=}=8}(\phi, \phi, \phi, \phi)-\frac{\mathcal{M}_{1}^{(-2)}(\sigma, \epsilon)}{b^{2} m_{1} m_{2} \pi \sigma}\left(\frac{b^{4} \pi}{4}\right)^{\epsilon} \frac{\Gamma(1-2 \epsilon)}{\Gamma(\epsilon)} .
\end{aligned}
$$

$$
\begin{aligned}
& \left.\widetilde{\mathcal{M}_{1}^{N=8}}(\phi, \phi, \varphi, \varphi)\right|_{\mathrm{Qt} .} \\
& =\left.\widetilde{\mathcal{M}_{1}^{N=}}(\phi, \phi, \phi, \phi)\right|_{\mathrm{Qt} .}-\frac{\mathcal{M}_{1}^{(-2)}(\sigma, \epsilon)}{b^{2} m_{1} m_{2} \pi \sigma}\left(\frac{b^{4} \pi}{4}\right)^{\epsilon} \frac{\Gamma(1-2 \epsilon)}{\Gamma(\epsilon)} .
\end{aligned}
$$

The effect of the helicity choice on the only amplitude only affects the quantum part of the amplitude not the classical part so that

\section{Two-loop amplitude}

For the two-loop amplitude

$$
\begin{aligned}
\widetilde{\mathcal{M}_{2}^{N=}=8}(\phi, \phi, \phi, \phi)= & -\frac{1}{6}\left(\widetilde{\mathcal{M}_{0}^{N=}=8}(\phi, \phi, \phi, \phi)\right)^{3}+\left.i \widetilde{\mathcal{M}_{0}^{N=}=8}(\phi, \phi, \phi, \phi) \widetilde{\mathcal{M}_{1}^{N=}}(\phi, \phi, \phi, \phi)\right|_{\mathrm{Cl}} . \\
& +i \widetilde{\mathcal{M}_{0}^{N=}}(\phi, \phi, \phi, \phi)\left|\widetilde{\mathcal{M}_{1}^{N=}}(\phi, \phi, \phi, \phi)\right|_{\mathrm{Qt} .}+\left.\widetilde{\mathcal{M}_{2}^{N=}}(\phi, \phi, \phi, \phi)\right|_{\mathrm{Cl} .},
\end{aligned}
$$

with the classical piece at two-loop given by

$$
\begin{aligned}
\left.\widetilde{\mathcal{M}_{2}^{N=8}}(\phi, \phi, \phi, \phi)\right|_{\mathrm{Cl} .}= & \frac{16 G_{N}^{3} m_{1}^{2} m_{2}^{2} \sigma^{4}\left(\pi b^{2} e^{\gamma_{E}}\right)^{3 \epsilon}}{\hbar b^{2}\left(\sigma^{2}-1\right)^{\frac{5}{2}}}\left[-\left(\sigma^{2}-1\right)^{\frac{3}{2}} \operatorname{arccosh}(\sigma)\right. \\
& -\frac{i}{\pi \epsilon}\left(\frac{-1}{4\left(\sigma^{2}-1\right)}\right)^{\epsilon}\left((1+2 \epsilon) \sigma^{2} \sqrt{\sigma^{2}-1}+\sigma\left(\sigma^{2}-2\right) \operatorname{arccosh}(\sigma)\right. \\
& \left.+\epsilon\left(\left(\sigma^{2}-1\right)^{\frac{3}{2}}-\sigma\left(\sigma^{2}-2\right)\right) \operatorname{arccosh}^{2}(\sigma)-\epsilon \sigma\left(\sigma^{2}-2\right) \operatorname{Li}_{2}\left(2-2 \sigma\left(\sigma+\sqrt{\sigma^{2}-1}\right)\right)+\mathcal{O}(\epsilon)\right] .
\end{aligned}
$$

The contribution $i \widetilde{\mathcal{M}_{0}^{N}=8}(\phi, \phi, \phi, \phi)\left|\widetilde{\mathcal{M}_{1}^{N=}}(\phi, \phi, \phi, \phi)\right|_{\mathrm{Qt}}$. is of $1 / \hbar$ order as the classical contribution. But we have separated it off from the classical contribution because only Eq. (87) will contribute to the classical scattering angle, as will be shown in Sec. III C.

For the choice of helicity in $[11,15]$ with the kinematic factor in Eq. (55) we have

$$
\widetilde{\mathcal{M}_{2}^{N=8}}(\phi, \phi, \varphi, \varphi)=\widetilde{\mathcal{M}_{2}^{N=8}}(\phi, \phi, \phi, \phi)-\left(\frac{b^{6} \pi}{16}\right)^{\epsilon} \frac{\mathcal{M}_{2}^{(-2)}(\sigma)}{\hbar b^{2} m_{1} m_{2} \pi \sigma} \frac{\Gamma(1-3 \epsilon)}{\Gamma(2 \epsilon)} .
$$

The extra classical piece generated by the $|q|^{2}$ term in the kinematic factor in Eq. (55) goes into the modification of the quantum one-loop part as given in Eq. (84) so that the two-loop amplitude is decomposed as

$$
\begin{aligned}
\widetilde{\mathcal{M}_{2}^{N=}=8}(\phi, \phi, \varphi, \varphi)= & -\frac{1}{6}\left(\widetilde{\mathcal{M}_{0}^{N}=8}(\phi, \phi, \varphi, \varphi)\right)^{3}+\left.i \widetilde{\mathcal{M}_{0}^{N=}=8}(\phi, \phi, \phi, \phi) \widetilde{\mathcal{M}_{1}^{N=}=8}(\phi, \phi, \varphi, \varphi)\right|_{\mathrm{Cl}} . \\
& +i \widetilde{\mathcal{M}_{0}^{N=8}}(\phi, \phi, \phi, \phi)\left|\widetilde{\mathcal{M}_{1}^{N=}}(\phi, \phi, \varphi, \varphi)\right|_{\mathrm{Qt}}+\left.\widetilde{\mathcal{M}_{2}^{N=}}(\phi, \phi, \varphi, \varphi)\right|_{\mathrm{Cl} .},
\end{aligned}
$$

with

$$
\left.\widetilde{\mathcal{M}_{2}^{N=8}}(\phi, \phi, \varphi, \varphi)\right|_{\mathrm{Cl} .}=\left.\widetilde{\mathcal{M}_{2}^{N=8}}(\phi, \phi, \phi, \phi)\right|_{\mathrm{Cl} .}
$$

Since the classical part of the two-loop amplitude does not depend on the helicity of the external state we just set

$$
\mathcal{M}_{2}^{\mathrm{Cl}} .\left.(\sigma, b) \equiv \widetilde{\mathcal{M}_{2}^{N=8}}(\phi, \phi, \varphi, \varphi)\right|_{\mathrm{Cl} .}
$$

The classical contribution to the two-loop amplitude is composed of a real and imaginary part

$$
\mathcal{M}_{2}^{\mathrm{Cl}}(\sigma, b)=\mathcal{M}_{2}^{\mathrm{Cl} . \mathrm{R}}(\sigma, b)+i \mathcal{M}_{2}^{\mathrm{Cl}} \mathrm{I}(\sigma, b)+\mathcal{O}(1)
$$

The real part of the two-loop classical amplitude is free of divergences 


$$
\begin{aligned}
\mathcal{M}_{2}^{\text {Cl. R }}(\sigma, b) & =\operatorname{Re}\left(\mathcal{M}_{2}^{\mathrm{Cl} .}(\sigma, b)\right) \\
& =\left.\mathcal{M}_{2}^{\text {Cl. R }}(\sigma, b)\right|_{\mathrm{Cons}}+\left.\mathcal{M}_{2}^{\mathrm{Cl} . \mathrm{R}}(\sigma, b)\right|_{\mathrm{Rad}},
\end{aligned}
$$

and composed of a conservative part

$$
\begin{aligned}
\left.\mathcal{M}_{2}^{\text {Cl. R }}(\sigma, b)\right|_{\text {Cons }}= & -\frac{8 G_{N}^{3} m_{1}^{2} m_{2}^{2} \sigma^{4}}{b^{2}} \frac{\operatorname{arccosh}(\sigma)}{\sigma^{2}-1} \\
& +\mathcal{O}(\epsilon)
\end{aligned}
$$

and a radiation-reaction part

$$
\begin{aligned}
\left.\mathcal{M}_{2}^{\mathrm{Cl} . \mathrm{R}}(\sigma, b)\right|_{\mathrm{Rad}} & \\
= & \frac{8 G_{N}^{3} m_{1}^{2} m_{2}^{2} \sigma^{4}}{b^{2}\left(\sigma^{2}-1\right)^{\epsilon}}\left(\frac{\pi b^{2} e^{\gamma_{E}}}{4}\right)^{\epsilon} \\
& \times\left(\frac{\sigma^{2}}{\left(\sigma^{2}-1\right)^{2}}+\frac{\sigma\left(\sigma^{2}-2\right)}{\left(\sigma^{2}-1\right)^{\frac{5}{2}}} \operatorname{arccosh}(\sigma)\right)+\mathcal{O}(\epsilon) .
\end{aligned}
$$

The imaginary part of the scattering phase is given by

$$
\begin{aligned}
\mathcal{M}_{2}^{\text {Cl. I }}(\sigma, b)= & \operatorname{Im}\left(\mathcal{M}_{2}^{\text {Cl. }}(\sigma, b)\right) \\
= & -\frac{\left.\mathcal{M}_{2}^{\mathrm{Cl}} \cdot \mathrm{R}(\sigma, b)\right|_{\mathrm{Rad}}}{\pi \epsilon}+\frac{8 G_{N}^{3} m_{1}^{2} m_{2}^{2} \sigma^{4}}{\pi b^{2}\left(\sigma^{2}-1\right)^{\epsilon}}\left(\frac{\pi b^{2} e^{\gamma_{E}}}{4}\right)^{\epsilon} \\
& \times\left[-\frac{2 \sigma}{\left(\sigma^{2}-1\right)^{2}}+\frac{\sigma^{2}-2}{\left(\sigma^{2}-1\right)^{\frac{5}{2}}} \mathrm{Li}_{2}\left(2-2 \sigma\left(\sigma+\sqrt{\sigma^{2}-1}\right)\right)+\left(\frac{1}{\sigma\left(\sigma^{2}-1\right)}+\frac{\sigma^{2}-2}{\left(\sigma^{2}-1\right)^{\frac{5}{2}}}\right) \operatorname{arccosh}(\sigma)^{2}\right] \\
& +O(\epsilon) .
\end{aligned}
$$

This result establishes the relation conjectured in [15] between the radiation-reaction part of the real part and the infrared divergence of imaginary part

$$
\left.\lim _{\epsilon \rightarrow 0} \mathcal{M}_{2}^{\mathrm{Cl} . \mathrm{R}}(\sigma, b)\right|_{\mathrm{Rad}}=-\lim _{\epsilon \rightarrow 0} \epsilon \pi \mathcal{M}_{2}^{\mathrm{Cl} . \mathrm{I}}(\sigma, b) .
$$

At high-energy $\sigma \gg 1$ we have that

$$
\begin{aligned}
- & \frac{2 \sigma}{\left(\sigma^{2}-1\right)^{2}}+\frac{\sigma^{2}-2}{\left(\sigma^{2}-1\right)^{\frac{5}{2}}} \operatorname{Li}_{2}\left(2-2 \sigma\left(\sigma+\sqrt{\sigma^{2}-1}\right)\right) \\
& +\left(\frac{1}{\sigma\left(\sigma^{2}-1\right)}+\frac{\sigma^{2}-2}{\left(\sigma^{2}-1\right)^{\frac{5}{2}}}\right) \operatorname{arccosh}(\sigma)^{2} \\
= & -\frac{\zeta(2)+2}{\sigma^{3}}+O\left(\sigma^{-2}\right),
\end{aligned}
$$

and

$\frac{\sigma}{\left(\sigma^{2}-1\right)^{2}}+\frac{\sigma^{2}-2}{\left(\sigma^{2}-1\right)^{\frac{5}{2}}} \operatorname{arccosh}(\sigma)=\frac{1+\log (2 \sigma)}{\sigma^{3}}+O\left(\sigma^{-2}\right)$,

therefore by expanding the factor of $\left(\sigma^{2}-1\right)^{-\epsilon}=$ $1-\epsilon \log \left(\sigma^{2}-1\right)$ we have that the radiation part reads for $\sigma \gg 1$

$$
\begin{aligned}
\lim _{\sigma \gg 1} \mathcal{M}_{2}^{\mathrm{Cl}}(\sigma, b) \simeq & \left(1+\frac{i}{\pi}\left(-\frac{1}{\epsilon}+\log \left(\sigma^{2}-1\right)\right)\right) \\
& \times \operatorname{coeff}_{\epsilon^{0}}\left(\left.\mathcal{M}_{2}^{\mathrm{Cl} . \mathrm{R}}(\sigma, b)\right|_{\mathrm{Rad}}\right) .
\end{aligned}
$$

This shows that the $\log \left(\sigma^{2}-1\right)$ of Eq. (3.2) in [15] arises from the $\epsilon$ expansion of the $\left(\sigma^{2}-1\right)^{-\epsilon}$ in Eq. (96).

The soft and potential region. We now consider the limits $\sigma \rightarrow 1$ and $\epsilon \rightarrow 0$ of the result.

(i) In the limit $\epsilon \rightarrow 0$ with $\sigma$ fixed

$$
\begin{aligned}
\lim _{\epsilon \rightarrow \frac{0}{\sigma} \text { fixed }} \operatorname{Re}\left(\mathcal{M}_{2}^{\mathrm{Cl}}\right) & \\
= & \frac{8 \pi G_{N}^{3} m_{1}^{2} m_{2}^{2} \sigma^{4}}{b^{2}}\left(\frac{\sigma^{2}}{\left(\sigma^{2}-1\right)^{2}}\right. \\
& \left.+\operatorname{arccosh}(\sigma)\left(\sigma \frac{\sigma^{2}-2}{\left(\sigma^{2}-1\right)^{\frac{5}{2}}}-\frac{1}{\sigma^{2}-1}\right)\right),
\end{aligned}
$$

we recover the result from the soft region [16].

(ii) Whereas in the $\sigma \rightarrow 1$ limit with $\epsilon<0$ fixed we have

$$
\begin{aligned}
\lim _{\substack{\sigma \rightarrow 1 \\
\epsilon<0 \text { fixed }}} \operatorname{Re}\left(\mathcal{M}_{2}^{\mathrm{Cl}}\right) \\
=\frac{8 G_{N}^{3} m_{1}^{2} m_{2}^{2} \sigma^{4}}{b^{2}}\left(-\frac{\operatorname{arccosh}(\sigma)}{\left(\sigma^{2}-1\right)}\right. \\
\left.\quad+\frac{5}{3\left(\sigma^{2}-1\right)}+O(\sigma-1)^{0}\right)+O(\epsilon) .
\end{aligned}
$$

The leading contribution when $\sigma \rightarrow 1$ is the conservative piece computed in [12] whereas the subleading piece is the radiation-reaction contribution $[15,20]$. 


\section{The eikonal phase}

The full scattering matrix in the $b$ space can be expanded as [33]

$$
\begin{aligned}
1+i \mathcal{T}\left(\lambda_{1}, \ldots, \lambda_{4}\right) & =1+i \sum_{L \geq 0} \widetilde{\mathcal{M}_{L}^{N=8}}\left(\lambda_{1}, \ldots, \lambda_{4}\right) \\
& =\left(1+i 2 \Delta\left(\lambda_{1}, \ldots, \lambda_{4}\right)\right) e^{\frac{i 2 \delta(\sigma, b)}{\hbar}},
\end{aligned}
$$

where $\widetilde{\mathcal{M}_{L}^{N}=8}\left(\lambda_{1}, \ldots, \lambda_{4}\right)$ are the $L$-loop amplitudes, $\delta$ is the classical eikonal and $\Delta$ a quantum correction. In perturbative expansion we have

$\delta=\delta_{0}+\delta_{1}+\delta_{2}+\cdots ; \quad \Delta=\Delta_{1}+\Delta_{2}+\cdots$,

with $\delta_{r}$ and $\Delta_{r}$ are of order $G_{N}^{r+1}$, gives to the first order studied explicitly in this work

$$
\begin{aligned}
2 \delta_{L} & =\hbar \operatorname{Re}\left(\left.\widetilde{\mathcal{M}_{L}}\right|_{\mathrm{Cl}}\right), \quad L=0,1,2, \\
2 \Delta_{1} & =\left.\widetilde{\mathcal{M}_{1}}\right|_{\text {Qt. }}, \\
2 \Delta_{2} & =-\left.i \widetilde{\mathcal{M}_{0}} \widetilde{\mathcal{M}_{1}}\right|_{\text {Qt. }}+\operatorname{Im}\left(\left.\widetilde{\mathcal{M}_{2}}\right|_{\mathrm{Cl} .}\right) .
\end{aligned}
$$

The exponentiation of the perturbative expansion $1+$ $i \sum_{L} \mathcal{M}_{L}(\sigma, b)$ is only possible if the partial amplitudes $\mathcal{M}_{L}^{(r)}(\sigma, \epsilon)$ satisfy relations similar to the one noticed at one-loop in Eq. (80) and two-loop in Eq. (86). In particular the exponentiation is possible because the contributions more singular than the classical one in the $\hbar \rightarrow 0$ limit do satisfy unitarity related relations in $b$ space [27].

We have noticed before that the different choices of helicity made in [12] and [11,15] do not affect the classical part of the eikonal phase, and therefore $\delta$ is the same for all helicity choices as expected from the universality of classical gravitational interactions. Only the quantum part $\Delta$ depends on the helicity choice. A similar dependence helicity dependence of the external states on the quantum part of the one-loop amplitude was noticed in [34].

At tree level $L=0$ and one-loop $L=1$ orders the classical piece of the amplitude is real, and the eikonal phase is equal to the classical part of the amplitude. The classical part of the two-loop $L=2$ amplitude has an imaginary part (that was discussed above). We have decided to only exponentiate the real part of the classical two-loop amplitude since an imaginary contribution to the eikonal phase would violate unitarity. But this would have to be confirmed by an higher loop computation.
Therefore, the classical scattering angle is obtained by the stationary phase from the (real) eikonal phase

$$
\sin \left(\frac{\chi}{2}\right)=-\frac{\sqrt{s}}{m_{1} m_{2} \sqrt{\sigma^{2}-1}} \frac{\partial \delta(\sigma, b)}{\partial b},
$$

and does not depend on the helicity choice. Universality of the high-energy limit of gravitational scattering up to twoloop order was first demonstrated in Ref. [35].

\section{The first post-Minkowskian order}

Because the tree-level amplitude is independent of the helicity choice the scattering phase is given by the $2 \delta_{0}=$ $\hbar \times(78)$ therefore the scattering angle at the first postMinkowskian order is given by

$$
\begin{aligned}
\left.\sin \left(\frac{\chi}{2}\right)\right|_{1 P M} & =\frac{2 G_{N} \sqrt{s} \sigma^{2}}{b\left(\sigma^{2}-1\right)}(b \sqrt{\pi})^{2 \epsilon} \Gamma(1-\epsilon), \\
& =\frac{2 G_{N} \sqrt{s} \sigma^{2}}{b\left(\sigma^{2}-1\right)}+\mathcal{O}(\epsilon) .
\end{aligned}
$$

\section{The second post-Minkowskian order}

Because the helicity choice does not affect the classical piece of the one-loop amplitude, the one-loop scattering phase is given by $2 \delta_{1}=\hbar \times(81)$ leading to the scattering angle

$$
\begin{aligned}
\left.\sin \left(\frac{\chi}{2}\right)\right|_{2 P M}= & \frac{8 G_{N}^{2} \sqrt{s} \sigma^{4}\left(m_{1}+m_{2}\right)}{\left(\sigma^{2}-1\right)^{2} b} \\
& \times \frac{\left(2 b^{2} \pi\right)^{2 \epsilon} \Gamma\left(\frac{3}{2}-2 \epsilon\right) \Gamma\left(\frac{1}{2}-\epsilon\right)}{\Gamma(-\epsilon)} \\
= & 0+\mathcal{O}(\epsilon)
\end{aligned}
$$

At the leading order in $\epsilon$ the scattering angle at the second post-Minkowskian order vanishes. A result which has been linked in [30] to the nontriangle property of maximal supergravity amplitude [36].

The $\Delta_{1}$ is given by the leading quantum part of the oneloop amplitude. The result for the helicity choice in [12] and in $[11,15]$ differ by the shift in Eq. (83).

\section{The third post-Minkowskian order}

The two-loop order scattering phase is given by $2 \delta_{2}=$ $\hbar \times \operatorname{Re}(87)$ which does not depend on the helicity configuration as explained before

$$
\begin{aligned}
\delta_{2}(\sigma, b)= & \operatorname{Re}\left(\frac { 8 G _ { N } ^ { 3 } m _ { 1 } ^ { 2 } m _ { 2 } ^ { 2 } \sigma ^ { 4 } ( \pi b ^ { 2 } e ^ { \gamma _ { E } } ) ^ { 3 \epsilon } } { b ^ { 2 } ( \sigma ^ { 2 } - 1 ) ^ { \frac { 5 } { 2 } } } \left[-\left(\sigma^{2}-1\right)^{\frac{3}{2}} \operatorname{arccosh}(\sigma)-\frac{i}{\pi \epsilon}\left(\frac{-1}{4\left(\sigma^{2}-1\right)}\right)^{\epsilon}\left((1+2 \epsilon) \sigma^{2} \sqrt{\sigma^{2}-1}+\sigma\left(\sigma^{2}-2\right) \operatorname{arccosh}(\sigma)\right.\right.\right. \\
& \left.\left.+\epsilon\left(\left(\sigma^{2}-1\right)^{\frac{3}{2}}-\sigma\left(\sigma^{2}-2\right)\right) \operatorname{arccosh}^{2}(\sigma)-\epsilon \sigma\left(\sigma^{2}-2\right) \mathrm{Li}_{2}\left(2-2 \sigma\left(\sigma+\sqrt{\sigma^{2}-1}\right)\right)+O(\epsilon)\right]\right)
\end{aligned}
$$


The scattering angle at the third post-Minkowskian order is then given by

$$
\left.\sin \left(\frac{\chi}{2}\right)\right|_{3 P M}=-\frac{\sqrt{s}}{m_{1} m_{2} \sqrt{\sigma^{2}-1}} \frac{\partial\left(\delta_{2}\right)}{\partial b}
$$

and reads

$$
\left.\sin \left(\frac{\chi}{2}\right)\right|_{3 P M}=\frac{16 G_{N}^{3} \sqrt{s} m_{1} m_{2} \sigma^{4}}{b^{3}\left(\sigma^{2}-1\right)}\left(-\frac{\operatorname{arccosh}(\sigma)}{\sqrt{\sigma^{2}-1}}+\frac{1}{\left(4\left(\sigma^{2}-1\right)\right)^{\epsilon}}\left(\frac{\sigma\left(\sigma^{2}-2\right) \operatorname{arccosh}(\sigma)}{\left(\sigma^{2}-1\right)^{2}}+\frac{\sigma^{2}}{\left(\sigma^{2}-1\right)^{\frac{3}{2}}}\right)\right)+\mathcal{O}(\epsilon),
$$

which presents a conservative part in the first line and a radiation-reaction part, given in the second line.

We will see below how the $\epsilon$ expansion of the soft factor $\left(\sigma^{2}-1\right)^{-\epsilon}$ matches with the results of [15].

Using the angular momentum

$$
J=\frac{m_{1} m_{2} \sqrt{\sigma^{2}-1}}{\sqrt{s}} b \cos \left(\frac{\chi}{2}\right)
$$

we can decompose the scattering angle at the third postMinkowskian order

$$
\chi_{3 P M}=\chi_{3 P M}^{\mathrm{Schw}}+\chi_{3 P M}^{\mathrm{Cons}}+\chi_{3 P M}^{\mathrm{Rad}},
$$

into a Schwarzschild metric contribution

$$
\chi_{3 P M}^{\mathrm{Schw}}=-16 m_{1}^{3} m_{2}^{3} G_{N}^{3} \frac{\sigma^{6}}{3 J^{3}\left(\sigma^{2}-1\right)^{\frac{3}{2}}},
$$

a conservative part

$$
\chi_{3 P M}^{\text {Cons }}=-32 m_{1}^{4} m_{2}^{4} \sigma^{4} G_{N}^{3} \frac{\operatorname{arccosh}(\sigma)}{J^{3} s},
$$

and a radiation part

$$
\begin{aligned}
\chi_{3 P M}^{\mathrm{Rad}}= & 32 m_{1}^{4} m_{2}^{4} G_{N}^{3} \frac{\sigma^{4}}{J^{3} s} \frac{1}{\left(4\left(\sigma^{2}-1\right)\right)^{\epsilon}} \\
& \times\left(\frac{\sigma\left(\sigma^{2}-2\right) \operatorname{arccosh}(\sigma)}{\left(\sigma^{2}-1\right)^{\frac{3}{2}}}+\frac{\sigma^{2}}{\sigma^{2}-1}\right) .
\end{aligned}
$$

When setting $\left(\sigma^{2}-1\right)^{-\epsilon}=1$ these results reproduce Eq. (4.7) of [11]. We remark that taking the limit $\sigma \rightarrow 1$ with fixed $J$ gives $\chi_{3 P M}^{\mathrm{Rad}}=0$ because $\epsilon<0$.

\section{THE EVALUATION OF THE TWO-LOOP SCALAR DOUBLE BOXES}

In this section we provide details about the evaluation of the double-box leading to the results in Sec. III A 3. The two-loop amplitude in Eq. (69) after the Kaluza-Klein reduction in Eq. (54) reads

$$
\begin{aligned}
\mathcal{M}^{2-\operatorname{loop}}\left(p_{1}, p_{2}, p_{1}^{\prime}, p_{2}^{\prime}\right) & \\
= & \left(8 \pi G_{N}\right)^{3}\left(4 m_{1}^{2} m_{2}^{2} \sigma^{2}\left(J_{s}+J_{u}\right)\right. \\
& \left.+2 \hbar^{2} m_{1} m_{2}|\underline{\vec{q}}|^{2} \sigma J_{u}+\hbar^{4}|\underline{q}|^{4} J_{t}\right) .
\end{aligned}
$$

We see that the classical contribution arises from the contribution of order $1 / \hbar$ from $J_{s}+J_{u}$, from the contribution of order $1 / \hbar^{3}$ from $J_{u}$ and from the contribution of order $1 / \hbar^{5}$ from $J_{t}$.

We first give a summary of the results for the expansion of these contributions and discuss the comparison with the existing results in the literature. We will then gives details on the methods used for deriving these results.

\section{A. The $t$-channel contribution}

The $t$-channel contributions are given by the so-called $H$ diagrams integrals. Using the $q=|q| u_{q}$ and $q=\hbar \underline{q}$, by rescaling the loop integrations $\ell_{i} \rightarrow l_{i}|q|$ we have

$$
\begin{aligned}
J_{t}= & \frac{-|\underline{q}|^{2 D-12}}{2 \hbar^{5}} \int \frac{d^{D} l_{1} d^{D} l_{2}}{(2 \pi)^{2 D}}\left(\frac{1}{2 p_{1} \cdot l_{1}+i \varepsilon}-\frac{1}{2 p_{1}^{\prime} \cdot l_{1}-i \varepsilon}\right)\left(\frac{1}{2 p_{2} \cdot l_{2}-i \varepsilon}-\frac{1}{2 p_{2}^{\prime} \cdot l_{2}+i \varepsilon}\right) \\
& \times \frac{1}{\left(\left(l_{1}+u_{q}\right)^{2}+i \varepsilon\right)\left(\left(l_{2}+u_{q}\right)^{2}+i \varepsilon\right)\left(l_{1}^{2}+i \varepsilon\right)\left(l_{2}^{2}+i \varepsilon\right)\left(\left(l_{1}+l_{2}+u_{q}\right)^{2}+i \varepsilon\right)} .
\end{aligned}
$$


Because the integral is already of the order $1 / \hbar^{5}$ is it enough to keep the leading order in the $|q|$ expansion for extracting the classical piece ${ }^{5}$

$$
J_{t}=-\frac{|\underline{q}|^{-4-4 \epsilon}}{16 m_{1} m_{2} \hbar^{5} \epsilon^{4} \sqrt{\sigma^{2}-1}} \mathcal{I}_{6}(\sigma)+\mathcal{O}(\hbar|\underline{q}|),
$$

where $\mathcal{I}_{6}(\sigma)$ is one of the two-loop master integrals ${ }^{6}$ which is evaluated in Sec. VF; the final result is

$$
J_{t}=-\frac{1}{256 m_{1} m_{2} \hbar^{5} \pi^{3} \epsilon} \frac{\left(4 \pi e^{-\gamma_{E}}\right)^{2 \epsilon}}{|\underline{q}|^{4+4 \epsilon}} \frac{\operatorname{arccosh}(\sigma)}{\sqrt{\sigma^{2}-1}}\left(\pi+i\left(\frac{-1}{4\left(\sigma^{2}-1\right)}\right)^{\epsilon} \operatorname{arccosh}(\sigma)+\mathcal{O}(\epsilon)\right)+O(\hbar|\underline{q}|) .
$$

\section{B. The $u$-channel contribution}

For the $u$-channel contribution we have

$$
\begin{aligned}
J_{u}= & \frac{|q|^{2 D-10}}{96 \hbar^{3}} \int \frac{d^{D} l_{1} d^{D} l_{2}}{(2 \pi)^{D}} \frac{1}{\left(l_{1}^{2}+i \varepsilon\right)\left(l_{2}^{2}+i \varepsilon\right)\left(\left(l_{1}+l_{2}+u_{q}\right)^{2}+i \varepsilon\right)} \\
& \times \sum_{1 \leq i \neq j \leq i \leq i \neq k, j \neq n} \sum_{1 \leq k \neq n \leq 3} \frac{1}{\left(\bar{p}_{1} \cdot l_{i}+|\vec{q}| \frac{u_{q} \cdot l_{i}}{2}+i \varepsilon\right)\left(\bar{p}_{1} \cdot l_{j}-|\vec{q}| \frac{u_{q} \cdot l_{j}}{2}-i \varepsilon\right)} \\
& \times \frac{1}{\left(\bar{p}_{2} \cdot l_{k}-|\vec{q}| \frac{u_{q} \cdot l_{k}}{2}-i \varepsilon\right)\left(\bar{p}_{2} \cdot l_{n}+|\vec{q}| \frac{u_{q} \cdot l_{n}}{2}+i \varepsilon\right)} .
\end{aligned}
$$

We introduced the momenta $\bar{p}_{i}=p_{1}-q / 2$ and $\bar{p}_{2}=p_{2}+q / 2$ which are orthogonal to $q$. And as before we rescaled the loop momenta by $\ell_{i} \rightarrow l_{i}|q|$ with $q=u_{q}|q|$, and $q=\hbar \underline{q}$,

$$
\begin{aligned}
J_{u}^{0}= & \frac{|q|^{2 D-10}}{96 \hbar^{3}} \int \frac{d^{D} l_{1} d^{D} l_{2}}{(2 \pi)^{D}} \frac{1}{\left(l_{1}^{2}+i \varepsilon\right)\left(l_{2}^{2}+i \varepsilon\right)\left(\left(l_{1}+l_{2}+u_{q}\right)^{2}+i \varepsilon\right)} \\
& \times \sum_{1 \leq i \neq j \leq i} \sum_{1=k, j \neq n} \frac{1}{\left(\bar{p}_{1} \cdot l_{i}+i \varepsilon\right)\left(\bar{p}_{1} \cdot l_{j}-i \varepsilon\right)\left(\bar{p}_{2} \cdot l_{k}-i \varepsilon\right)\left(\bar{p}_{2} \cdot l_{n}+i \varepsilon\right)} .
\end{aligned}
$$

Using the principal part formula in Eq. (18), we can rewrite this expression using delta functions

$$
\begin{aligned}
J_{u}^{0}= & \frac{|q|^{2 D-10}}{192 \hbar^{3}} \int \frac{d^{D} l_{1} d^{D} l_{2}}{(2 \pi)^{2 D-4}} \frac{\delta\left(\overline{p_{1}} \cdot l_{1}\right) \delta\left(\overline{p_{1}} \cdot l_{2}\right) \delta\left(\overline{p_{2}} \cdot l_{1}\right) \delta\left(\overline{p_{2}} \cdot l_{2}\right)}{l_{1}^{2} l_{2}^{2}\left(l_{1}+l_{2}+u_{q}\right)^{2}} \\
& -\frac{|\underline{q}|^{2 D-10}}{64 \hbar^{3}} \int \frac{d^{D} l_{1} d^{D} l_{2}}{(2 \pi)^{2 D-2}} \frac{\delta\left(\overline{p_{1}} \cdot l_{1}\right) \delta\left(\overline{p_{2}} \cdot l_{2}\right)}{l_{1}^{2} l_{2}^{2}\left(l_{1}+l_{2}+u_{q}\right)^{2}}\left(\frac{1}{\bar{p}_{1} \cdot l_{2}-i \varepsilon}+\frac{1}{\bar{p}_{1} \cdot l_{2}+i \varepsilon}\right)\left(\frac{1}{\bar{p}_{2} \cdot l_{1}-i \varepsilon}+\frac{1}{\bar{p}_{2} \cdot l_{1}+i \varepsilon}\right),
\end{aligned}
$$

which can be expressed using the master integral $\mathcal{I}_{9}(\sigma)$ as

$$
J_{u}^{0}=\frac{|\underline{q}|^{-2-4 \epsilon}}{16 \hbar^{3} m_{1}^{2} m_{2}^{2}\left(\sigma^{2}-1\right) \epsilon^{4}}\left(\mathcal{I}_{9}(\sigma)-b_{9} \epsilon^{2}\right) .
$$

Because the integral is of order $1 / \hbar^{3}$ one needs the leading order expansion of the integral for getting the classical contribution

\footnotetext{
${ }^{5}$ The power of $\hbar$ arises because this diagram as two massive propagators and five massless propagators giving a power of $\hbar$, to be $3 L+1-(2+2(3 L-1))=1-3 L=-5$ for $L=2$. But the total amplitude in Eq. (117) has a power $\hbar^{4}$ in the numerator.

${ }^{6}$ In the Appendix we explain how to convert the double-box integrals to the integrals with a generalized propagator that is used in the definition of the master integrals.
} 


$$
J_{u}=\frac{|\underline{q}|^{-2-4 \epsilon}}{16 \hbar^{3} m_{1}^{2} m_{2}^{2}\left(\sigma^{2}-1\right) \epsilon^{4}}\left(\mathcal{I}_{9}(\sigma)-b_{9} \epsilon^{2}\right)+O(\hbar|\underline{q}|)
$$

where $\mathcal{I}_{9}(\sigma)$ is one of the two-loop master integrals evaluated in Sec. V G and $b_{9}$ is the constant of integration determined in Eq. (189). At the leading order in $|q|$ in $D=4-2 \epsilon$ we get that

$$
\begin{aligned}
J_{u}= & \frac{i\left(4 \pi e^{-\gamma_{E}}\right)^{2 \epsilon}}{512 \pi^{3} \hbar^{3} m_{1}^{2} m_{2}^{2}|q|^{2+4 \epsilon}\left(\sigma^{2}-1\right) \epsilon^{2}}\left(\frac{-1}{4\left(\sigma^{2}-1\right)}\right)^{\epsilon} \\
& \times\left(\operatorname{arccosh}(\sigma)-\epsilon\left(\operatorname{arccosh}^{2}(\sigma)+\operatorname{Li}_{2}\left[2-2 \sigma\left(\sigma+\sqrt{\sigma^{2}-1}\right)\right]\right)\right)+O\left(\epsilon^{0}\right) .
\end{aligned}
$$

\section{The $(s+u)$-channel contribution}

The contribution of the combined $s$ and $u$ channels is simplified thanks to symmetrization, and reads

$$
\begin{aligned}
J_{s-u}:=J_{s}+J_{u}= & \frac{|\underline{q}|^{2 D-10}}{96 \hbar^{3}} \int \frac{d^{D} l_{1} d^{D} l_{2}}{(2 \pi)^{2 D}} \frac{1}{\left(l_{1}^{2}+i \varepsilon\right)\left(l_{2}^{2}+i \varepsilon\right)\left(\left(l_{1}+l_{2}+u_{q}\right)^{2}+i \varepsilon\right)} \\
& \times \sum_{1 \leq i \neq j \leq 3} \sum_{1 \leq k \neq n \leq 3} \frac{1}{\left(\overline{p_{1}} \cdot l_{i}+|\vec{q}| \frac{u_{q} \cdot l_{i}}{2}+i \varepsilon\right)\left(\overline{p_{1}} \cdot l_{j}-|\vec{q}| \frac{u_{q} \cdot l_{j}}{2}-i \varepsilon\right)} \\
& \times \frac{1}{\left(\overline{p_{2}} \cdot l_{k}-|\vec{q}| \frac{u_{q} \cdot l_{k}}{2}-i \varepsilon\right)\left(\overline{p_{2}} \cdot l_{n}+|\vec{q}| \frac{u_{q} \cdot l_{n}}{2}+i \varepsilon\right)} .
\end{aligned}
$$

We introduced the momenta $\bar{p}_{i}=p_{1}-q / 2$ and $\bar{p}_{2}=p_{2}+$ $q / 2$ which are orthogonal to $q$. And as before we rescaled the loop momenta by $\ell_{i} \rightarrow l_{i}|q|$ with $q=u_{q}|q|$, and $q=\hbar \underline{q}$.

In this expression we have neglected the $l_{i}^{2}$ terms in the denominators in the sum for the reason that they do not contribute to the classical limit. In the $\hbar$ expansion the $l_{i}^{2}$ contribution will cancel a massless propagator and give a reduced graph that does not have a classical limit, in a similar way as we have seen when expanding Eq. (32).
Because the integral is of order $1 / \hbar^{3}$ one needs to expand the denominator in the integral to the second order in $\hbar^{2}$ for getting the classical contribution

$$
J_{s-u}=J_{s-u}^{0}+\hbar|\underline{q}| J_{s-u}^{1}+\hbar^{2}|\underline{q}|^{2} J_{s-u}^{2}+O\left(|\vec{q}|^{3}\right) .
$$

\section{Evaluation of $J_{s-u}^{0}$}

The leading order expansion in $|q|$ of the double-box contribution $J_{s-u}$ is given by

$$
J_{s-u}^{0}=\frac{|q|^{2 D-10}}{96 \hbar^{3}} \int \frac{d^{D} l_{1} d^{D} l_{2}}{(2 \pi)^{2 D}} \sum_{1 \leq i \neq j \leq 3} \sum_{1 \leq k \neq n \leq 3} \frac{1}{\left(\overline{p_{1}} \cdot l_{i}+i \varepsilon\right)\left(\overline{p_{1}} \cdot l_{j}-i \varepsilon\right)\left(\overline{p_{2}} \cdot l_{k}-i \varepsilon\right)\left(\overline{p_{2}} \cdot l_{n}+i \varepsilon\right) l_{1}^{2} l_{2}^{2}\left(l_{1}+l_{2}+u_{q}\right)^{2}},
$$

which can be rewritten in terms of delta functions as

$$
J_{s-u}^{0}=\frac{|q|^{2 D-10}}{96 \hbar^{3}} \int \frac{d^{D} l_{1} d^{D} l_{2}}{(2 \pi)^{2 D-4}} \frac{\delta\left(\overline{p_{1}} \cdot l_{1}\right) \delta\left(\overline{p_{1}} \cdot l_{2}\right) \delta\left(\overline{p_{2}} \cdot l_{1}\right) \delta\left(\overline{p_{2}} \cdot l_{2}\right)}{l_{1}^{2} l_{2}^{2}\left(l_{1}+l_{2}+u_{q}\right)^{2}}
$$

and evaluated to

$$
J_{s-u}^{0}=-\frac{|q|^{2 D-10}}{96 \hbar^{3}} \frac{1}{\left(\bar{p}_{1} \cdot \bar{p}_{2}\right)^{2}-\bar{p}_{1}^{2} \bar{p}_{2}^{2}} \int \frac{d^{D-2} \vec{l}_{1} d^{D-2} \vec{l}_{2}}{(2 \pi)^{2 D-4}} \frac{1}{\vec{l}_{1}^{2} \vec{l}_{2}^{2}\left(\vec{l}_{1}+\vec{l}_{2}+\vec{u}_{q}\right)^{2}},
$$

using that $\left(\bar{p}_{1} \cdot \bar{p}_{2}\right)^{2}-\bar{p}_{1}^{2} \bar{p}_{2}^{2}=m_{1}^{2} m_{2}^{2}\left(\sigma^{2}-1\right)-\frac{|\vec{q}|^{2} s}{4}$ and the integrals in Appendix A of [24] give that 


$$
J_{s-u}^{0}=-\frac{|q|^{2 D-10}}{96 \hbar^{3}} \frac{\Gamma\left(\frac{D}{2}-2\right)^{3} \Gamma(5-D)}{(4 \pi)^{D-2} \Gamma\left(\frac{3(D-4)}{2}\right)\left(m_{1}^{2} m_{2}^{2}\left(\sigma^{2}-1\right)-\hbar^{2} \frac{|q|^{2} s}{4}\right)} .
$$

Expanding the denominator in $|\vec{q}|$ gives a contribution to the classical order

$$
J_{s-u}^{0}=-\frac{|q|^{2 D-10}}{96 \hbar^{3}} \frac{\Gamma\left(\frac{D}{2}-2\right)^{3} \Gamma(5-D)}{(4 \pi)^{D-2} \Gamma\left(\frac{3(D-4)}{2}\right) m_{1}^{2} m_{2}^{2}\left(\sigma^{2}-1\right)}-\frac{|q|^{2 D-8}}{384 \hbar} \frac{\Gamma\left(\frac{D}{2}-2\right)^{3} \Gamma(5-D)}{(4 \pi)^{D-2} \Gamma\left(\frac{3(D-4)}{2}\right) m_{1}^{4} m_{2}^{4}\left(\sigma^{2}-1\right)^{2}}+O\left(|q|^{2 D-6}\right) .
$$

The first term of order $1 / \hbar^{3}$ is the superclassical contribution, whereas the next term of order $1 / \hbar$ is a classical contribution:

$$
J_{s-u}^{0}=-\frac{1}{384 \mid \underline{q}^{4 \epsilon+2} \hbar^{3}} \frac{\Gamma(-\epsilon)^{3} \Gamma(1+2 \epsilon)}{(4 \pi)^{2-2 \epsilon} \Gamma(-3 \epsilon)} \frac{4 m_{1}^{2} m_{2}^{2}\left(\sigma^{2}-1\right)+|\vec{q}|^{2} s}{m_{1}^{4} m_{2}^{4}\left(\sigma^{2}-1\right)^{2}} .
$$

\section{The evaluation of $J_{s-u}^{1}$}

The subleading order expansion in $|q|$ of the double-box contribution $J_{s-u}$ is given by

$$
\begin{aligned}
\hbar|\underline{q}| J_{s-u}^{1}= & -\frac{|\underline{q}|^{2 D-9}}{96 \hbar^{2}} \int \frac{d^{D} l_{1} d^{D} l_{2}}{(2 \pi)^{2 D-3}} \frac{3 i\left(1-2 u_{q} \cdot l_{1}\right) \delta\left(\overline{p_{1}} \cdot l_{1}\right) \delta\left(\overline{p_{2}} \cdot l_{1}\right) \delta\left(\overline{p_{2}} \cdot l_{2}\right)}{2\left(\overline{p_{1}} \cdot l_{2}\right)^{2} l_{1}^{2} l_{2}^{2}\left(l_{1}+l_{2}+u_{q}\right)^{2}} \\
& -\frac{|\underline{q}|^{2 D-9}}{96 \hbar^{2}} \int \frac{d^{D} l_{1} d^{D} l_{2}}{(2 \pi)^{2 D-3}} \frac{3 i\left(1-2 u_{q} \cdot l_{1}\right) \delta\left(\overline{p_{1}} \cdot l_{1}\right) \delta\left(\overline{p_{1}} \cdot l_{2}\right) \delta\left(\overline{p_{2}} \cdot l_{1}\right)}{2\left(\overline{p_{2}} \cdot l_{2}\right)^{2} l_{1}^{2} l_{2}^{2}\left(l_{1}+l_{2}+u_{q}\right)^{2}} .
\end{aligned}
$$

This integral is of order $|q|^{2 D-9} / \hbar^{2}$ and imaginary. The $|q|^{2}$ expansion cannot lead to a contribution to the classical part of the amplitude of order $|q|^{2 D-8} / \hbar$. So this integral will not contribute to the classical result but to the super-classical one.

The integral $J_{s-u}^{1}$ is

$$
\hbar|\underline{q}| J_{s-u}^{1}=\frac{i\left(m_{1}+m_{2}\right)|\underline{q}|^{-1-4 \epsilon}}{512 \hbar^{2} \pi^{\frac{5}{2}} m_{1}^{3} m_{2}^{3}\left(\sigma^{2}-1\right)^{\frac{3}{2}}} \frac{(4 \pi)^{2 \epsilon} \Gamma\left(\frac{1}{2}-\epsilon\right)^{2} \Gamma\left(\frac{1}{2}+2 \epsilon\right) \Gamma(-\epsilon) \Gamma\left(\frac{1}{2}-2 \epsilon\right)}{\Gamma\left(\frac{1}{2}-3 \epsilon\right) \Gamma(-2 \epsilon)},
$$

and will not contribute to the classical limit.

\section{The evaluation of $J_{s-u}^{2}$}

The contribution at the order $O\left(|q|^{2}\right)$ is decomposed into several pieces (each one being an integral with two $\delta$ functions) that we treat in order

$$
\hbar^{2}|\underline{q}|^{2} J_{s-u}^{2}=J_{\triangleright}^{2}+J_{\square}^{2, \perp}+J_{\triangleleft}^{2}+J_{\square}^{2, \|} .
$$

(i) $J_{\triangleright}^{2}$ is expanded on the master integrals of Sec. V using LITERED

$$
J_{\triangleright}^{2}=\frac{|q|^{-4 \epsilon}}{64 m_{1}^{2} m_{2}^{4}\left(\sigma^{2}-1\right)^{2} \hbar} \lim _{\sigma \rightarrow 1}\left(\frac{2}{3} \frac{\mathcal{I}_{2}(\sigma)}{2 \epsilon^{4} \sqrt{\sigma^{2}-1}}-\frac{3+4 \epsilon}{3} \frac{\mathcal{I}_{9}^{+-}(\sigma)}{\epsilon^{4}}\right),
$$

using the results in Sec. V E we obtain

$$
\begin{aligned}
J_{\triangleright}^{2} & =\frac{|q|^{-4 \epsilon}}{64 m_{1}^{2} m_{2}^{4}\left(\sigma^{2}-1\right)^{2} \hbar}\left(\frac{b_{4}}{3 \epsilon}-\frac{(3+4 \epsilon) b_{9}^{+-}}{3 \epsilon^{2}}\right) \\
& =\left(\frac{4 \pi e^{-\gamma_{E}}}{|\underline{q}|^{2}}\right)^{2 \epsilon} \frac{1+\epsilon}{2048 \pi^{2} \epsilon^{2} m_{1}^{2} m_{2}^{4}\left(\sigma^{2}-1\right)^{2} \hbar}+\mathcal{O}\left(\epsilon^{0}\right) .
\end{aligned}
$$


(ii) The contribution $J_{\triangleleft}^{2}$ is obtained by exchanging $m_{1}$ and $m_{2}$ and is given by

$$
J_{\triangleleft}^{2}=\left(\frac{4 \pi e^{-\gamma_{E}}}{|\underline{q}|^{2}}\right)^{2 \epsilon} \frac{1+\epsilon}{2048 \pi^{2} \epsilon^{2} m_{1}^{4} m_{2}^{2}\left(\sigma^{2}-1\right)^{2} \hbar}+\mathcal{O}\left(\epsilon^{0}\right) .
$$

(iii) The decomposition of $J_{\square}^{2, \perp}$ on the master integrals with LITERED is given by

$$
\begin{aligned}
J_{\square}^{2, \perp}= & -\frac{|\underline{q}|^{-4 \epsilon}}{64 m_{1}^{3} m_{2}^{3}\left(\sigma^{2}-1\right)^{2} \hbar}\left(-\frac{8 \sqrt{\sigma^{2}-1}}{\epsilon^{2}} \mathcal{I}_{2}(\sigma)+\frac{2(1+2 \epsilon) \sqrt{\sigma^{2}-1}}{\epsilon^{3}} \mathcal{I}_{3}(\sigma)\right. \\
& \left.-\frac{2(1+6 \epsilon) \sigma}{3 \epsilon^{3}} \mathcal{I}_{4}(\sigma)-\frac{(3+2 \epsilon) \sigma}{3 \epsilon^{4}}\left(\mathcal{I}_{9}^{+-}(\sigma)+\mathcal{I}_{9}^{++}(\sigma)\right)\right),
\end{aligned}
$$

using the result from the evaluation of the master integrals in Sec. V we get

$$
\begin{aligned}
J_{\square}^{2, \perp}= & \frac{-|q|^{-4 \epsilon}}{64 m_{1}^{3} m_{2}^{3}\left(\sigma^{2}-1\right)^{2} \hbar \epsilon^{2}}\left(\frac{-\left(2 \epsilon b_{4}+(3+2 \epsilon)\left(b_{9}^{+-}+b_{9}^{++}\right)\right) \sigma}{3}+2(1+2 \epsilon) b_{3}\left(\sigma^{2}-1\right)^{\frac{1}{2}-\epsilon}\right. \\
& \left.-2 \sigma b_{3} \int_{1}^{\sigma} \frac{d t}{\left(t^{2}-1\right)^{\frac{1}{2}+\epsilon}}\right)+\mathcal{O}\left(\epsilon^{0}\right) .
\end{aligned}
$$

(iv) For $J_{\square}^{2, \|}$ we have

$$
\begin{aligned}
J_{\square}^{2, \|}= & -\frac{|q|^{-4 \epsilon}}{256 \hbar} \int \frac{d^{D} l_{1} d^{D} l_{2}}{(2 \pi)^{2 D-2}} \frac{\left(1-2 u_{q} \cdot l_{1}\right)^{2} \delta\left(\overline{p_{1}} \cdot l_{1}\right) \delta\left(\overline{p_{2}} \cdot l_{1}\right)}{l_{1}^{2} l_{2}^{2}\left(l_{1}+l_{2}+u_{q}\right)^{2}} \\
& \times\left(\frac{1}{\left(\overline{p_{1}} \cdot l_{2}-i \varepsilon\right)^{2}\left(\overline{p_{2}} \cdot l_{2}+i \varepsilon\right)^{2}}+\frac{1}{\left(\overline{p_{1}} \cdot l_{2}+i \varepsilon\right)^{2}\left(\overline{p_{2}} \cdot l_{2}+i \varepsilon\right)^{2}}\right),
\end{aligned}
$$

which evaluates to

$$
J_{\square}^{2, \|}=\left(\frac{4 \pi e^{-\gamma_{E}}}{|q|^{2}}\right)^{2 \epsilon} \frac{i(1+2 \epsilon)\left(\sigma \operatorname{arccosh}(\sigma)-\sqrt{\sigma^{2}-1}-\frac{i \pi \sigma}{2}\right)}{1024 \epsilon^{2} \pi^{3} m_{1}^{3} m_{2}^{3}\left(\sigma^{2}-1\right)^{2} \hbar}+\mathcal{O}\left(\epsilon^{0}\right) .
$$

Summing all these contributions and using that for $\epsilon<0$ and $\sigma>1^{7}$

$$
\begin{aligned}
\int_{1}^{\sigma} \frac{d t}{\left(t^{2}-1\right)^{\frac{1}{2}+\epsilon}} & =4^{\epsilon} \int_{1}^{\sigma+\sqrt{\sigma^{2}-1}} \frac{x^{-1+2 \epsilon}}{\left(x^{2}-1\right)^{2 \epsilon}} d x \\
& =\frac{1}{\left(\sigma^{2}-1\right)^{\epsilon}}\left(\operatorname{arccosh}(\sigma)-\epsilon\left(\operatorname{arccosh}(\sigma)^{2}+\operatorname{Li}_{2}\left(2-2 \sigma\left(\sigma+\sqrt{\sigma^{2}-1}\right)\right)\right)+\mathcal{O}(\epsilon)\right)
\end{aligned}
$$

we have

$$
\begin{aligned}
\hbar^{2} \underline{q}^{2} J_{s-u}^{2}= & \left(\frac{4 \pi e^{-\gamma_{E}}}{|\underline{q}|^{2}}\right)^{2 \epsilon} \frac{1}{2048 \epsilon^{2} \pi^{3} m_{1}^{3} m_{2}^{3}\left(\sigma^{2}-1\right)^{2} \hbar}\left(\frac{(1+\epsilon) \pi s}{m_{1} m_{2}}+2 i(1+2 \epsilon)\left(\sigma \operatorname{arccosh}(\sigma)-\sqrt{\sigma^{2}-1}\right)\right. \\
& +2 i\left(\frac{-1}{4\left(\sigma^{2}-1\right)}\right)^{\epsilon}\left(\sigma\left(\operatorname{arccosh}(\sigma)-\epsilon\left(\operatorname{arccosh}^{2}(\sigma)+\operatorname{Li}_{2}\left(2-2 \sigma\left(\sigma+\sqrt{\sigma^{2}-1}\right)\right)\right)\right)\right. \\
& \left.\left.\left.-(1+2 \epsilon) \sqrt{\sigma^{2}-1}\right)\right)\right)+\mathcal{O}\left(\epsilon^{0}\right) .
\end{aligned}
$$

\footnotetext{
${ }^{7}$ We recall that $\operatorname{arccosh}(\sigma)=\log \left(\sigma+\sqrt{\sigma^{2}-1}\right)$ for $\sigma>1$.
} 


\section{The final expression for $J_{s-u}$}

The sum of all the contributions is given by

$$
\begin{aligned}
J_{s-u}= & \frac{1}{(4 \pi)^{4} \hbar}\left(\frac{4 \pi e^{-\gamma_{E}}}{|\underline{q}|^{2}}\right)^{2 \epsilon}\left(-\frac{\pi^{2}}{2 \epsilon^{2} \hbar^{2}|\underline{q}|^{2} m_{1}^{2} m_{2}^{2}\left(\sigma^{2}-1\right)}+\frac{i \pi(1+2 \epsilon)\left(\sigma \operatorname{arccosh} \sigma-\sqrt{\sigma^{2}-1}\right)}{4 \epsilon^{2} m_{1}^{3} m_{2}^{3}\left(\sigma^{2}-1\right)^{2}}+\frac{\pi^{2} s}{8 \epsilon m_{1}^{4} m_{2}^{4}\left(\sigma^{2}-1\right)^{2}}\right. \\
& +\frac{i \pi}{4 \epsilon^{2} m_{1}^{3} m_{2}^{3}\left(\sigma^{2}-1\right)^{2}}\left(\frac{-1}{4\left(\sigma^{2}-1\right)}\right)^{\epsilon}\left(\sigma\left(\operatorname{arccosh}(\sigma)-\epsilon\left(\operatorname{arccosh}^{2}(\sigma)+\operatorname{Li}_{2}\left(2-2 \sigma\left(\sigma+\sqrt{\sigma^{2}-1}\right)\right)\right)\right)\right. \\
& \left.\left.\left.\left.-(1+2 \epsilon) \sqrt{\sigma^{2}-1}\right)\right)\right)\right)+\mathcal{O}\left(\epsilon^{0}\right),
\end{aligned}
$$

we recall that the $1 / \hbar$ contribution is of order $\epsilon$ and does not contribute to this order.

\section{DIFFERENTIAL EQUATIONS FOR THE MASTER INTEGRALS}

For the computation of the two-loop integrals we need to evaluate the following nine master integrals

$$
\begin{gathered}
\mathcal{I}_{1}(\sigma)=2 \epsilon^{4} \mathcal{I}^{0,0,1,1,1,1,0}, \\
\mathcal{I}_{2}(\sigma)=2 \epsilon^{4} \sqrt{\sigma^{2}-1} \mathcal{I}^{0,0,0,0,1,1,1}, \\
\mathcal{I}_{3}(\sigma)=2 \epsilon^{3} \sqrt{\sigma^{2}-1} \mathcal{I}^{0,0,0,0,1,1,2},
\end{gathered}
$$

$\mathcal{I}_{4}(\sigma)=4 \epsilon^{2}\left(\sigma^{2}-1\right) \mathcal{I}^{-1,-1,0,0,1,1,3}+\epsilon^{2}(1+2 \epsilon) \sigma \mathcal{I}^{0,0,0,0,1,1,2}$,

$\mathcal{I}_{7}(\sigma)=8 \epsilon^{4}\left(\sigma^{2}-1\right) \mathcal{I}^{-1,-1,1,1,1,1,1}+4 \epsilon^{4} \sigma \mathcal{I}^{0,0,1,1,1,1,0}$,

$$
\mathcal{I}_{8}(\sigma)=-\epsilon^{3} \mathcal{I}^{0,1,0,0,1,1,2},
$$$$
\mathcal{I}_{9}(\sigma)=\epsilon^{4} \mathcal{I}^{1,1,0,0,1,1,1},
$$

with the following definition for the master integral

$$
\begin{aligned}
\mathcal{I}^{n_{1}, n_{2}, n_{3}, n_{4}, n_{5}, n_{6}, n_{7}} \equiv & \int \frac{d^{D-1} l_{1} d^{D-1} l_{2}}{(2 \pi)^{2 D-2}} \frac{1}{\left(k \cdot l_{1}\right)^{n_{1}}\left(k \cdot l_{2}\right)^{n_{2}}\left(l_{1}^{2}\right)^{n_{3}}\left(\left(u_{q}+l_{2}\right)^{2}\right)^{n_{4}}\left(\left(l_{1}-u_{q}\right)^{2}\right)^{n_{5}}} \\
& \times \frac{1}{\left(l_{2}^{2}\right)^{n_{6}}\left(\left(l_{1}+l_{2}\right)^{2}-2(\sigma-1) k \cdot l_{1} k \cdot l_{2}\right)^{n_{7}}},
\end{aligned}
$$

where we have defined $k^{2} \equiv u_{q}^{2} \equiv-1$ and $k \cdot u_{q} \equiv 0$.

In the Appendix we explain how to convert the two-loop integrals in $D$ dimensions with delta-function insertions in the numerator to two-loop integrals with a generalized propagator in $D-1$ dimensions.

Using LITERED [25] we find the following differential system of equations

$$
\frac{d}{d \sigma}\left(\begin{array}{l}
\mathcal{I}_{1}(\sigma) \\
\mathcal{I}_{2}(\sigma) \\
\mathcal{I}_{3}(\sigma) \\
\mathcal{I}_{4}(\sigma) \\
\mathcal{I}_{5}(\sigma) \\
\mathcal{I}_{6}(\sigma) \\
\mathcal{I}_{7}(\sigma) \\
\mathcal{I}_{8}(\sigma) \\
\mathcal{I}_{9}(\sigma)
\end{array}\right)=\epsilon\left(\begin{array}{ccccccccc}
0 & 0 & 0 & 0 & 0 & 0 & 0 & 0 & 0 \\
0 & \frac{6 \sigma}{\sigma^{2}-1} & 0 & \frac{1}{\sqrt{\sigma^{2}-1}} & 0 & 0 & 0 & 0 & 0 \\
0 & 0 & -\frac{2 \sigma}{\sigma^{2}-1} & \frac{2}{\sqrt{\sigma^{2}-1}} & 0 & 0 & 0 & 0 & 0 \\
0 & -\frac{12}{\sqrt{\sigma^{2}-1}} & -\frac{2}{\sqrt{\sigma^{2}-1}} & 0 & 0 & 0 & 0 & 0 & 0 \\
0 & 0 & 0 & 0 & -\frac{2 \sigma}{\sigma^{2}-1} & 0 & 0 & 0 & 0 \\
0 & 0 & -\frac{4 \sigma}{\sigma^{2}-1} & -\frac{2}{\sqrt{\sigma^{2}-1}} & -\frac{4 \sigma}{\sigma^{2}-1} & -\frac{2 \sigma}{\sigma^{2}-1} & \frac{2}{\sqrt{\sigma^{2}-1}} & 0 & 0 \\
-\frac{4}{\sigma^{2}-1} & -\frac{12}{\sqrt{\sigma^{2}-1}} & -\frac{8}{\sqrt{\sigma^{2}-1}} & 0 & -\frac{8}{\sqrt{\sigma^{2}-1}} & -\frac{2}{\sqrt{\sigma^{2}-1}} & \frac{2 \sigma}{\sigma^{2}-1} & 0 & 0 \\
0 & 0 & 0 & 0 & 0 & 0 & 0 & 0 & 0 \\
0 & 0 & \frac{1}{\sqrt{\sigma^{2}-1}} & 0 & 0 & 0 & 0 & 0 & 0
\end{array}\right)\left(\begin{array}{c}
\mathcal{I}_{1}(\sigma) \\
\mathcal{I}_{2}(\sigma) \\
\mathcal{I}_{3}(\sigma) \\
\mathcal{I}_{4}(\sigma) \\
\mathcal{I}_{5}(\sigma) \\
\mathcal{I}_{6}(\sigma) \\
\mathcal{I}_{7}(\sigma) \\
\mathcal{I}_{8}(\sigma) \\
\mathcal{I}_{9}(\sigma)
\end{array}\right) .
$$


For the resolution of this system of differential equation one must pay attention to the fact that the $\epsilon \rightarrow 0$ limit and $\sigma \rightarrow 1$ limit do not commute. Solving the differential system in an $\epsilon$ expansion for fixed $\sigma$ does not lead to the correct answer at $\sigma=1 .^{8}$ We solve the differential system using iterated integrals methods and numerical methods and use PYSECDEC [38] for validating our analysis.

A. The master integrals $\mathcal{I}_{1}(\sigma), \mathcal{I}_{5}(\sigma)$, and $\mathcal{I}_{8}(\sigma)$

The following integrals are easily integrated

$$
\begin{gathered}
\mathcal{I}_{1}(\sigma)=b_{1} \epsilon^{4}, \\
\mathcal{I}_{5}(\sigma)=b_{5} \epsilon\left(\sigma^{2}-1\right)^{-\epsilon}, \\
\mathcal{I}_{8}(\sigma)=a_{8},
\end{gathered}
$$

where $b_{1}, b_{5}$, and $a_{8}$ are constants of integrations that will be determined later.

When deriving the expression for the master integrals we will keep the factors $\left(\sigma^{2}-1\right)^{-\epsilon}$ explicit in order to keep a control of the limits $\epsilon \rightarrow 0$ and $\sigma \rightarrow 1$. When solving this system of differential equation we assume that $\epsilon<0$.

B. The master integrals $\mathcal{I}_{\mathbf{2}}(\sigma), \mathcal{I}_{\mathbf{3}}(\sigma)$, and $\mathcal{I}_{\mathbf{4}}(\sigma)$

We remark that the equation for $\mathcal{I}_{2}(\sigma)$

$$
\frac{d \mathcal{I}_{2}(\sigma)}{d \sigma}=\frac{6 \epsilon \sigma}{\sigma^{2}-1} \mathcal{I}_{2}(\sigma)+\frac{\epsilon}{\sqrt{\sigma^{2}-1}} \mathcal{I}_{4}(\sigma),
$$

leads to

$\mathcal{I}_{2}(\sigma)=a_{2}\left(\sigma^{2}-1\right)^{3 \epsilon}+\epsilon\left(\sigma^{2}-1\right)^{3 \epsilon} \int_{1}^{\sigma} \frac{\mathcal{I}_{4}(t)}{\left(t^{2}-1\right)^{\frac{1}{2}+3 \epsilon}} d t$.

Using the mean value theorem $[39, \S \mathrm{V} .4]$ the integral can be written as

$$
\left(\sigma^{2}-1\right)^{3 \epsilon} \int_{1}^{\sigma} \frac{\mathcal{I}_{4}(t)}{\left(t^{2}-1\right)^{\frac{1}{2}+3 \epsilon}} d t=\left(\sigma^{2}-1\right)^{1+3 \epsilon} \frac{\mathcal{I}_{4}(t(\sigma))}{\left(t(\sigma)^{2}-1\right)^{\frac{1}{2}+3 \epsilon}},
$$

where $t(\sigma) \in[1, \sigma]$, and we deduce that the integral vanishes in the limit $\sigma \rightarrow 1$. For this one needs that $\mathcal{I}_{4}(\sigma)$ stays finite which is confirmed by an numerical evaluation with PYSECDEC.

We impose the regularity of $\mathcal{I}_{2}(\sigma)$ at $\sigma=1$, which implies that $a_{2}=0$ therefore

\footnotetext{
${ }^{8}$ This phenomenon has already been noticed in other contexts and it has been illustrated in Sec. 7.3 of [37] for instance.
}

$$
\mathcal{I}_{2}(\sigma)=\epsilon\left(\sigma^{2}-1\right)^{3 \epsilon} \int_{1}^{\sigma} \frac{\mathcal{I}_{4}(t)}{\left(t^{2}-1\right)^{\frac{1}{2}+3 \epsilon}} d t
$$

In a similar fashion we have

$$
\begin{aligned}
& \mathcal{I}_{3}(\sigma)=b_{3} \epsilon\left(\sigma^{2}-1\right)^{-\epsilon}+2 \epsilon\left(\sigma^{2}-1\right)^{-\epsilon} \int_{1}^{\sigma} \frac{\mathcal{I}_{4}(t)}{\left(t^{2}-1\right)^{\frac{1}{2}-\epsilon}} d t, \\
& \mathcal{I}_{4}(\sigma)=a_{4}-12 \epsilon \int_{1}^{\sigma} \frac{\mathcal{I}_{2}(t)}{\left(t^{2}-1\right)^{\frac{1}{2}}} d t-2 \epsilon \int_{1}^{\sigma} \frac{\mathcal{I}_{3}(t)}{\left(t^{2}-1\right)^{\frac{1}{2}}} d t .
\end{aligned}
$$

This leads to the following $\epsilon$ expansion

$$
\begin{aligned}
\mathcal{I}_{2}(\sigma)= & \left(b_{4}\left(\sigma^{2}-1\right)^{3 \epsilon} \int_{1}^{\sigma} \frac{d t}{\left(t^{2}-1\right)^{\frac{1}{2}+3 \epsilon}}\right. \\
& \left.-2 b_{3}\left(\sigma^{2}-1\right)^{3 \epsilon} \int_{1}^{\sigma} \frac{d t_{2}}{\left(t_{2}^{2}-1\right)^{\frac{1}{2}+3 \epsilon}} \int_{1}^{t_{2}} \frac{d t_{1}}{\left(t_{1}^{2}-1\right)^{\frac{1}{2}+\epsilon}}\right) \epsilon^{3} \\
& +\mathcal{O}\left(\epsilon^{4}\right),
\end{aligned}
$$

and

$$
\begin{aligned}
\mathcal{I}_{3}(\sigma)= & \frac{b_{3} \epsilon}{\left(\sigma^{2}-1\right)^{\epsilon}} \\
& -\left(4 \frac{b_{3}}{\left(\sigma^{2}-1\right)^{\epsilon}} \int_{1}^{\sigma} \frac{d t_{2}}{\left(t_{2}^{2}-1\right)^{\frac{1}{2}-\epsilon}} \int_{1}^{t_{2}} \frac{d t_{1}}{\left(t_{1}^{2}-1\right)^{\frac{1}{2}+\epsilon}}\right. \\
& \left.-2 \frac{b_{4}}{\left(\sigma^{2}-1\right)^{\epsilon}} \int_{1}^{\sigma} \frac{d t}{\left(t^{2}-1\right)^{\frac{1}{2}-\epsilon}}\right) \epsilon^{3}+\mathcal{O}\left(\epsilon^{4}\right),
\end{aligned}
$$

and

$$
\mathcal{I}_{4}(\sigma)=\left(b_{4}-2 b_{3} \int_{1}^{\sigma} \frac{d t}{\left(t^{2}-1\right)^{\frac{1}{2}+\epsilon}}\right) \epsilon^{2}+\mathcal{O}\left(\epsilon^{4}\right) .
$$

\section{The master integrals $\mathcal{I}_{7}(\sigma)$ and $\mathcal{I}_{9}(\sigma)$}

Then $\mathcal{I}_{9}$ is obtained by

$$
\begin{aligned}
\mathcal{I}_{9}(\sigma) & =b_{9} \epsilon^{2}+\epsilon \int_{1}^{\sigma} \frac{\mathcal{I}_{3}(t)}{\sqrt{t^{2}-1}} d t \\
& =\left(b_{9}+b_{3} \int_{1}^{\sigma} \frac{d t}{\left(t^{2}-1\right)^{\frac{1}{2}+\epsilon}}\right) \epsilon^{2}+\mathcal{O}\left(\epsilon^{4}\right) .
\end{aligned}
$$

The integral $\mathcal{I}_{7}$ is given by

$$
\begin{aligned}
\mathcal{I}_{7}(\sigma) & =a_{7}\left(\sigma^{2}-1\right)^{\epsilon} \\
& -2 \epsilon\left(\sigma^{2}-1\right)^{\epsilon} \int_{1}^{\sigma} \frac{6 \mathcal{I}_{2}(t)+4 \mathcal{I}_{3}(t)+4 \mathcal{I}_{5}(t)+\mathcal{I}_{6}(t)}{\left(t^{2}-1\right)^{\frac{1}{2}+\epsilon}} d t .
\end{aligned}
$$


The regularity of the solution at $\sigma=1$ for $\epsilon<0$ imposes that $a_{7}=0$. Using the previous results and keeping only the terms up to $\mathcal{O}\left(\epsilon^{2}\right)$ gives

$\mathcal{I}_{7}(\sigma)=-8 \epsilon^{2}\left(b_{3}+b_{5}\right)\left(\sigma^{2}-1\right)^{\epsilon} \int_{1}^{\sigma} \frac{d t}{\left(t^{2}-1\right)^{\frac{1}{2}+2 \epsilon}}+\mathcal{O}\left(\epsilon^{3}\right)$.

\section{The master integrals $\mathcal{I}_{6}(\sigma)$}

For $\mathcal{I}_{6}$ we have

$$
\begin{aligned}
\mathcal{I}_{6}(\sigma)= & \frac{b_{6}}{\left(\sigma^{2}-1\right)^{\epsilon}}-\frac{4 \epsilon}{\left(\sigma^{2}-1\right)^{\epsilon}} \int_{1}^{\sigma} \frac{t\left(\mathcal{I}_{3}(t)+\mathcal{I}_{5}(t)\right)}{\left(t^{2}-1\right)^{1-\epsilon}} d t \\
& -\frac{2 \epsilon}{\left(\sigma^{2}-1\right)^{\epsilon}} \int_{1}^{\sigma} \frac{\mathcal{I}_{4}(t)-\mathcal{I}_{7}(t)}{\left(t^{2}-1\right)^{\frac{1}{2}-\epsilon}} d t .
\end{aligned}
$$

Up to the order $\mathcal{O}(\epsilon)^{4}$ leads to

$$
\begin{aligned}
\mathcal{I}_{6}(\sigma)= & \frac{b_{6}}{\left(\sigma^{2}-1\right)^{\epsilon}}-\frac{4 \epsilon^{2}\left(b_{3}+b_{5}\right)}{\left(\sigma^{2}-1\right)^{\epsilon}} \int_{1}^{\sigma} \frac{t d t}{t^{2}-1} \\
& -\frac{2 \epsilon}{\left(\sigma^{2}-1\right)^{\epsilon}} \int_{1}^{\sigma} \frac{\mathcal{I}_{4}(t)-\mathcal{I}_{7}(t)}{\left(t^{2}-1\right)^{\frac{1}{2}-\epsilon}} d t+\mathcal{O}(\epsilon)^{4} .
\end{aligned}
$$

The finiteness of this expression imposes that $b_{5}=-b_{3}$, so that

$$
\begin{aligned}
\mathcal{I}_{6}(\sigma)= & \left(\frac{b_{6}}{\left(\sigma^{2}-1\right)^{\epsilon}}-\frac{2 b_{4}}{\left(\sigma^{2}-1\right)^{\epsilon}} \int_{1}^{\sigma} \frac{d t}{\left(t^{2}-1\right)^{\frac{1}{2}-\epsilon}}\right. \\
& \left.+\frac{4 b_{3}}{\left(\sigma^{2}-1\right)^{\epsilon}} \int_{1}^{\sigma} \frac{d t_{2}}{\left(t_{2}^{2}-1\right)^{\frac{1}{2}-\epsilon}} \int_{1}^{t_{1}} \frac{d t_{1}}{\left(t_{1}^{2}-1\right)^{\frac{1}{2}+\epsilon}}\right) \epsilon^{3} \\
& +\mathcal{O}\left(\epsilon^{4}\right) .
\end{aligned}
$$

\section{E. Determination of the constants of integrations}

We determine the constants of integration so that we have a solution that is valid in all regimes and allows a control of the $\epsilon \rightarrow 0$ and $\sigma \rightarrow 1$ limits.

\section{1. $b_{1}$ constant}

Evaluating the $\mathcal{I}_{1}$ thanks to the results of the Appendix A of [24] we find

$$
\begin{aligned}
b_{1} & =\frac{1}{2}\left(\int \frac{d^{D-1} \vec{l}}{(2 \pi)^{D-1}} \frac{1}{\vec{l}^{2}\left(\vec{l}+\vec{u}_{q}\right)^{2}}\right)^{2} \\
& =\frac{\Gamma\left(\frac{1}{2}+\epsilon\right)^{2} \Gamma\left(\frac{1}{2}-\epsilon\right)^{4}}{2(4 \pi)^{3-2 \epsilon} \Gamma(1-2 \epsilon)^{2}} .
\end{aligned}
$$

\section{2. $b_{4}$ constant}

For $b_{4}$ we have in $D=4-2 \epsilon$

$$
\begin{aligned}
b_{4} & =\lim _{\sigma \rightarrow 1} \frac{\mathcal{I}_{2}(\sigma)}{\epsilon^{3} \sqrt{\sigma^{2}-1}} \\
& =2 \epsilon \int \frac{d^{D-1} l_{1} d^{D-1} l_{2}}{(2 \pi)^{2 D-2}} \frac{1}{l_{1}^{2} l_{2}^{2}\left(l_{1}+l_{2}+u_{q}\right)^{2}} \\
& =-\frac{2 \epsilon \Gamma\left(\frac{1}{2}-\epsilon\right)^{3} \Gamma(2 \epsilon)}{(4 \pi)^{D-1} \Gamma\left(\frac{3}{2}-3 \epsilon\right)} .
\end{aligned}
$$

So that at leading order in $\epsilon$,

$$
b_{4}=-\frac{\left(4 \pi e^{-\gamma_{E}}\right)^{2 \epsilon}}{32 \pi^{2}}+\mathcal{O}(\epsilon) .
$$

\section{3. $b_{3}$ and $b_{5}$ constants}

The constants $b_{3}$ and $b_{5}=-b_{3}$ cannot be determined from the $\sigma=1$ limit, but we can do a direct computation of $\mathcal{I}_{5}$ and check the result with numerical analysis using PYSECDEC [38]. We have using the definition of $\mathcal{I}_{5}{ }^{9}$

$$
\begin{aligned}
& \frac{\mathcal{I}_{5}(\sigma)}{2 \epsilon^{2}(4 \epsilon-1)(2 \epsilon-1)} \\
& =\frac{\mathcal{I}^{0,0,0,1,0,1,1}}{\sqrt{\sigma^{2}-1}} \\
& =\frac{m_{1} m_{2}}{\sqrt{\sigma^{2}-1}} \int \frac{d^{D} l_{1} d^{D} l_{2}}{(2 \pi)^{2 D-2}} \frac{\delta\left(\bar{p}_{1} \cdot l_{1}\right) \delta\left(\bar{p}_{2} \cdot l_{2}\right)}{l_{2}^{2}\left(l_{2}-u_{q}\right)^{2}\left(l_{1}+l_{2}\right)^{2}} .
\end{aligned}
$$

Then we write

$$
\delta\left(\overline{p_{1}} \cdot l_{1}\right)=\frac{1}{2 i \pi}\left(\frac{1}{\overline{p_{1}} \cdot l_{1}-i \varepsilon}-\frac{1}{\overline{p_{1}} \cdot l_{1}+i \varepsilon}\right)
$$

using the principal part identity in (18) and perform the $l_{1}$ integration using the results of the Appendix A of [24], giving

$$
\begin{aligned}
& { }^{9} \text { If one expands the master integral } \\
& \begin{aligned}
\mathcal{I}^{0,0,0,1,0,1,1} & =\int \frac{d^{D-1} l_{1} d^{D-1} l_{2}}{(2 \pi)^{2 D-2}} \\
& \times \frac{1}{\left(l_{2}+u_{q}\right)^{2} l_{2}^{2}\left(\left(l_{1}+l_{2}\right)^{2}-2(\sigma-1) k \cdot l_{1} k \cdot l_{2}\right)},
\end{aligned}
\end{aligned}
$$

in small $\sigma-1$ series and integrates terms by terms, then the result is 0 by dimension regularization of massless tadpoles. This is where we see the difference with the potential region computation in [12] which has $\mathcal{I}_{5}(\sigma)=0$. This would set $b_{5}=b_{3}=0$ in the computation and all radiation reactions will cancel. 


$$
\begin{aligned}
& \frac{\mathcal{I}_{5}(\sigma)}{2 \epsilon^{2}(4 \epsilon-1)(2 \epsilon-1)} \\
& =-\frac{4 \Gamma(1-\epsilon) \Gamma(-1+2 \epsilon)}{(4 \pi)^{2-\epsilon}\left(\sigma^{2}-1\right)^{\epsilon}} \int \frac{d^{D-1} \overrightarrow{l_{2}}}{(2 \pi)^{D-1}} \frac{\left(2 \vec{k} \cdot \overrightarrow{l_{2}}\right)^{1-2 \epsilon}}{\vec{l}_{2}^{2}\left(\vec{l}_{2}-\overrightarrow{u_{q}}\right)^{2}} .
\end{aligned}
$$

Using again the results of the Appendix A of [24] gives

$\mathcal{I}_{5}(\sigma)=-\frac{i \epsilon(4 \pi)^{2 \epsilon}}{32 \pi^{2}\left(\sigma^{2}-1\right)^{\epsilon}} \frac{(-1)^{\epsilon} \Gamma(1-2 \epsilon)^{2} \Gamma(1+2 \epsilon)^{2} \Gamma(1-\epsilon)}{\Gamma(1-4 \epsilon) \Gamma(1+\epsilon)}$,

with $(-1)^{\epsilon}=e^{i \pi \epsilon}=1+i \pi \epsilon+\mathcal{O}\left(\epsilon^{2}\right)$. So at the end we get

$$
b_{5}=-b_{3}=-\left(-\frac{1}{4}\right)^{\epsilon} \frac{i\left(4 \pi e^{-\gamma_{E}}\right)^{2 \epsilon}}{32 \pi^{3}}+\mathcal{O}\left(\epsilon^{2}\right) .
$$

We have confirmed this result using numerical evaluations with PYSECDEC [38].

\section{4. $a_{8}$ constant}

For the constant $a_{8}$ we have to take care of the $i \varepsilon$ prescription, defining

$a_{8}^{ \pm}=-\epsilon^{3} \int \frac{d^{D-1} l_{1} d^{D-1} l_{2}}{(2 \pi)^{2 D-2}} \frac{1}{\left(k \cdot l_{2} \pm i \varepsilon\right) l_{1}^{2} l_{2}^{2}\left(l_{1}+l_{2}+u_{q}\right)^{4}}$.

Using the results of the Appendix A of [24] gives

$a_{8}^{ \pm}= \pm \frac{i \sqrt{\pi} \epsilon^{3} \Gamma(-\epsilon) \Gamma\left(-\frac{1}{2}-2 \epsilon\right) \Gamma\left(\frac{3}{2}+2 \epsilon\right) \Gamma\left(\frac{1}{2}-\epsilon\right) \Gamma\left(-\frac{1}{2}-\epsilon\right)}{(4 \pi)^{3-2 \epsilon} \Gamma(-2 \epsilon) \Gamma\left(-\frac{1}{2}-3 \epsilon\right)}$.

So that we get in the end

$$
a_{8}^{ \pm}=\mp \frac{i \epsilon^{3}\left(4 \pi e^{-\gamma_{E}}\right)^{2 \epsilon}}{32 \pi}+\mathcal{O}\left(\epsilon^{4}\right)
$$

\section{5. $b_{9}$ constant}

Similarly for $b_{9}$ we have

$$
\begin{aligned}
b_{9}^{ \pm \pm}= & \epsilon^{2} \int \frac{d^{D-1} l_{1} d^{D-1} l_{2}}{(2 \pi)^{2 D-2}} \\
& \times \frac{1}{\left(k \cdot l_{1} \pm i \epsilon\right)\left(k \cdot l_{2} \pm i \epsilon\right) l_{1}^{2} l_{2}^{2}\left(l_{1}+l_{2}+u_{q}\right)^{2}} .
\end{aligned}
$$

Using the results of the Appendix A of [24] gives

$$
\begin{aligned}
b_{9}^{+-} & =b_{9}^{-+}=-\frac{\epsilon^{2}}{6} \frac{\Gamma(-\epsilon)^{3} \Gamma(1+2 \epsilon)}{(4 \pi)^{2-2 \epsilon} \Gamma(-3 \epsilon)} \\
& =-\frac{\left(4 \pi e^{-\gamma_{E}}\right)^{2 \epsilon}}{32 \pi^{2}}+\mathcal{O}\left(\epsilon^{2}\right),
\end{aligned}
$$

and

$$
b_{9}^{++}=b_{9}^{--}=-2 b_{9}^{+-} .
$$

\section{F. Final determination of the master integral $\mathcal{I}_{\mathbf{6}}(\sigma)$}

Using that the leading order expansion in $\epsilon \rightarrow 0$ (with $\epsilon<0)$ and $\sigma>1$

$$
\int_{1}^{\sigma} \frac{d t}{\left(t^{2}-1\right)^{\frac{1}{2}-\epsilon}}=\operatorname{arccosh}(\sigma)+O(\epsilon)
$$

and

$\int_{1}^{\sigma} \frac{d t_{2}}{\left(t_{2}^{2}-1\right)^{\frac{1}{2}}} \int_{1}^{t_{2}} \frac{d t_{1}}{\left(t_{1}^{2}-1\right)^{\frac{1}{2}}}=\frac{(\operatorname{arccosh}(\sigma))^{2}}{2}+O(\epsilon)$,

and using the values for the constant of integration $b_{4}$ in Eq. (177) and $b_{3}$ in Eq. (184) and that $b_{6}=0,{ }^{10}$ leads to

$$
\begin{aligned}
\mathcal{I}_{6}(\sigma)= & \frac{\left(4 \pi e^{-\gamma_{E}}\right)^{2 \epsilon} \epsilon^{3}}{8 \pi^{3}} \operatorname{arcsinh}\left(\sqrt{\frac{\sigma-1}{2}}\right) \\
& \left.\times\left(\pi+2 i\left(\frac{-1}{4\left(\sigma^{2}-1\right)}\right)\right)^{\epsilon} \operatorname{arcsinh}\left(\sqrt{\frac{\sigma-1}{2}}\right)\right) \\
& +\mathcal{O}\left(\epsilon^{4}\right) .
\end{aligned}
$$

\section{G. Final determination of the master integral $\mathcal{I}_{\mathbf{9}}(\boldsymbol{\sigma})$}

The master integral $\mathcal{I}_{9}(\sigma)$ in Eq. (170) reads

$$
\mathcal{I}_{9}(\sigma)-b_{9} \epsilon^{2}=b_{3} \epsilon^{2} \int_{1}^{\sigma} \frac{d t}{\left(t^{2}-1\right)^{\frac{1}{2}+\epsilon}}+\mathcal{O}\left(\epsilon^{4}\right)
$$

The constant of integration $b_{3}$ has been determined in Eq. (184) and $b_{9}$ in Eq. (189). Using the result in Eq. (145) for the integral we have

$$
\begin{aligned}
\mathcal{I}_{9}(\sigma)= & b_{9} \epsilon^{2}+\frac{b_{3} \epsilon^{2}}{\left(\sigma^{2}-1\right)^{\epsilon}}\left(\operatorname{arccosh}(\sigma)-\epsilon\left(\operatorname{arccosh}(\sigma)^{2}\right.\right. \\
& \left.\left.+\operatorname{Li}_{2}\left(2-2 \sigma\left(\sigma+\sqrt{\sigma^{2}-1}\right)\right)\right)+\mathcal{O}(\epsilon)\right) .
\end{aligned}
$$

\footnotetext{
${ }^{10}$ This has been confirmed by numerical evaluations using PYSECDEC [38].
} 
At high-energy $\sigma \gg 1$ we have

$$
\begin{aligned}
\mathcal{I}_{9}(\sigma) \simeq & b_{9} \epsilon^{2}+\frac{b_{3} \epsilon^{2}}{\left(\sigma^{2}-1\right)^{\epsilon}}\left(\log (2 \sigma)+\left(\zeta(2)+\log ^{2}(2 \sigma)\right) \epsilon\right) \\
& +O\left(\epsilon^{4}\right) .
\end{aligned}
$$

\section{CONCLUSION}

Motivated by the current interest in extending the computation of two-body interactions in general relativity to the post-Minkowskian regime, much effort has gone into establishing a systematic approach based on modern scattering amplitude techniques. Critical to this program is a complete understanding of how $S$-matrix calculations lead to classical scattering information.

We have here reanalyzed the evaluation of those integrals that contribute to classical observables in gravity. We have aimed to simplify and streamline the known method of soft region evaluation so as to make it amenable to high-order calculations. In the process of this program, we believe we have clarified issues related to apparent differences, both between potential-region and soft-region integrations and between soft-region integrations that use different boundary conditions. At one-loop order, our analysis confirms in a straightforward manner earlier results for both Einstein gravity and $\mathcal{N}=8$ supergravity. At two-loop order, we have focused exclusively on the simpler case of $\mathcal{N}=8$ supergravity. Our results here are in complete agreement with recent results by Di Vecchia, Heissenberg, Russo, and Veneziano [11,15,16]. In the process of comparison, we have made some observations that we hope will help clarify those computations.

While our method described here is firmly anchored in the method of integration in the soft region, it offers several advantages that will become further apparent at higher loop order. Most importantly, by adding sets of integrands we recover combinations that precisely generate delta functions, and hence effectively reduce the dimensionality of integrations. ${ }^{11}$ Besides, the combination of diagrams

\footnotetext{
${ }^{11}$ Combining terms in this manner introduces delta functions in an optimal way. This generalizes the well-known one-loop eikonal procedure of [40]. The same idea was also used to simplify the $H$ and crossed- $H$ diagrams in [12].
}

before integrating leads to a quite significant reduction in the number of master integrals that need to be evaluated.

In addition to the real terms of the amplitude that will exponentiate in the eikonal limit, we have also established in detail the relation between the real and imaginary part at two-loop order discussed in $[15,16]$. This seems to be a new situation where the cancellation of infrared divergences must happen at the linear level in the $S$ matrix rather than in quantum mechanical cross sections. This issue should be better clarified in future work.

\section{ACKNOWLEDGMENTS}

We would like to thank Samuel Abreu and Lorenzo Magnea for discussions. We thank Roman Lee for having giving us access to the version 2 of LITERED and answering questions about its usage. The research of P.V. has received funding from the ANR Grant "Amplitudes" No. ANR-17- CE31-0001-01, and the ANR Grant "SMAGP" No. ANR-20-CE40-0026-01 and is partially supported by Laboratory of Mirror Symmetry NRU HSE, RF Government Grant No. 14.641.31.0001. P.V. is grateful to the I.H.E.S. for the use of their computer resources. The work of P. H. D. was supported in part by DFF Grant No. 0135-00089A. The work of N.E. J. B.-B. was supported in part by the Carlsberg Foundation.

\section{APPENDIX: MAPPING TO INTEGRALS WITH A GENERALIZED PROPAGATOR}

In this Appendix we explain how one can convert the two-loop integrals with $\delta(\bar{p} \cdot l)$ insertions [coming from the application of the principle-part prescription in (18)] to integrals with a generalized propagator $1 /\left(\left(l_{1}+l_{2}+u_{q}\right)^{2}+\right.$ $\left.2(1-\sigma)\left(k \cdot l_{1}\right)\left(k \cdot l_{2}\right)\right)$ that we use for the determination of the master integrals in Sec. V.

Using these manipulations we have the following correspondence between the integrals. The dashed lines are massless propagators and the wavy lines are the generalized propagator. 

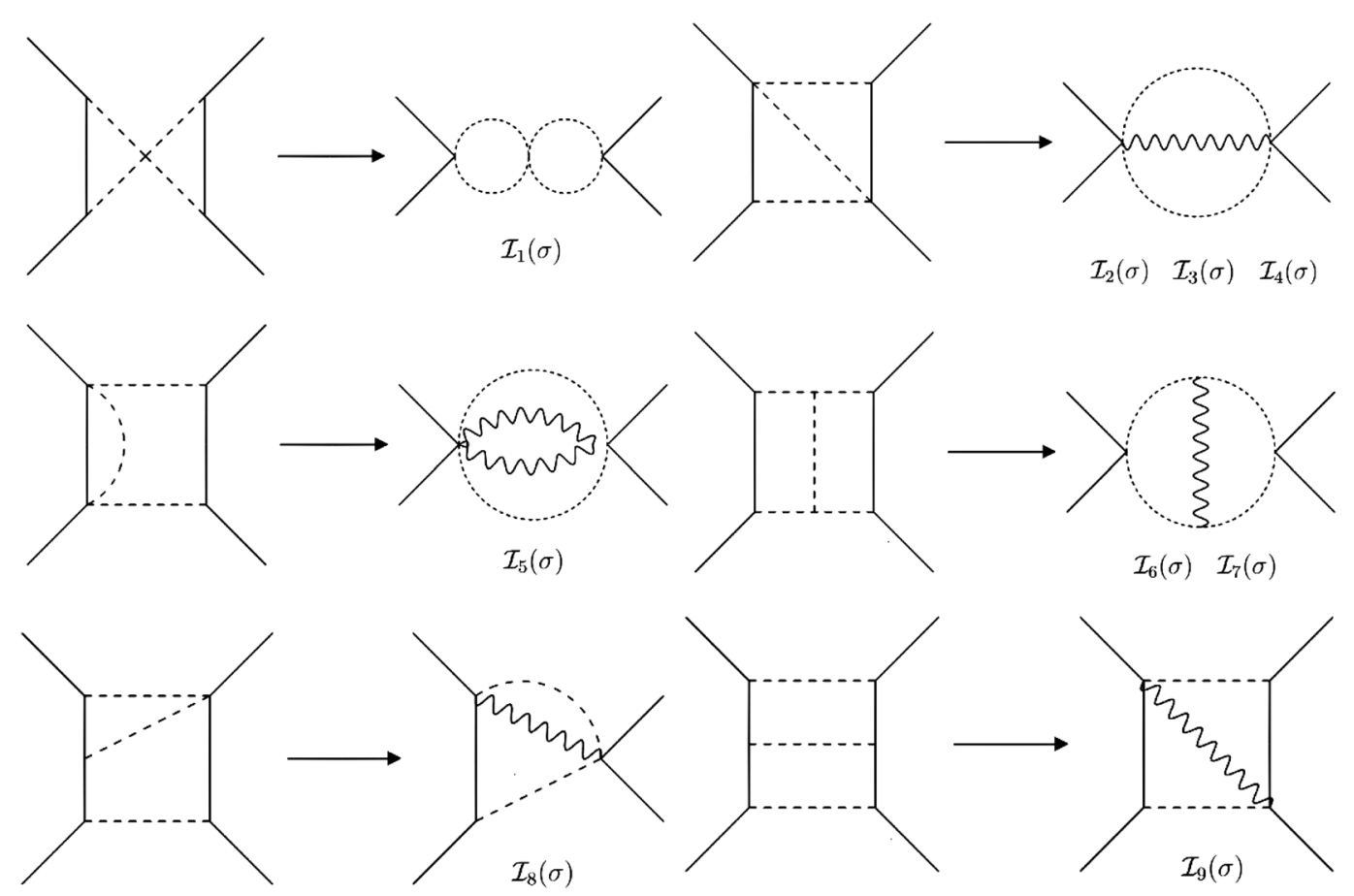

We start with the two-loop $D$-dimensional integral

$$
\begin{aligned}
\mathcal{D}= & \int \frac{d^{D} l_{1} d^{D} l_{2}}{(2 \pi)^{2 D-2}} \delta\left(\overline{p_{1}} \cdot l_{1}\right) \delta\left(\overline{p_{2}} \cdot l_{2}\right) \\
& \times \frac{1}{\left(\overline{p_{1}} \cdot l_{2} \pm i \varepsilon\right)^{n_{1}}\left(\overline{p_{2}} \cdot l_{1} \pm i \varepsilon\right)^{n_{2}}\left(\left(u_{q}+l_{1}\right)^{2}\right)^{n_{3}}\left(\left(u_{q}+l_{2}\right)^{2}\right)^{n_{4}}\left(l_{1}^{2}\right)^{n_{5}}\left(l_{2}^{2}\right)^{n_{6}}\left(\left(l_{1}+l_{2}+u_{q}\right)^{2}\right)^{n_{7}}} .
\end{aligned}
$$

We recall that $\bar{p}_{1} \cdot u_{q}=\bar{p}_{2} \cdot u_{q}=0$. Consider the vector $k_{1}=\frac{-m_{2} \sigma \bar{p}_{1}+m_{1} \bar{p}_{2}}{m_{1} m_{2} \sqrt{\sigma^{2}-1}}$. The vector $k_{1}$ is in the plane generated by $\bar{p}_{1}$ and $\bar{p}_{2}$, and we have $k_{1}^{2}=-1$ and $\bar{p}_{1} \cdot k_{1}=0$. We have also $\bar{p}_{2}^{\mu}=\frac{m_{2} \sigma}{m_{1}} \bar{p}_{1}^{\mu}+m_{2} \sqrt{\sigma^{2}-1} k_{1}^{\mu}$ and $\bar{p}_{2} \cdot k_{1}=-m_{2} \sqrt{\sigma^{2}-1}$. Separating the integration variables $l_{i}$ into $l_{i}=\tilde{l}_{i}+\hat{l}_{i}$, where $\tilde{l}_{i}$ is the components of $l_{i}$ inside the plane defined by $\left(p_{1}, p_{2}\right)$ and $\hat{l}_{i}$ are the other components of the $D-2$ space, orthogonal to $\left(\bar{p}_{1}, \bar{p}_{2}\right)$.

Note also that $\left(\frac{p_{1}}{m_{1}}, k_{1}\right)$ forms an orthogonal basis of the two-dimensional space spanned by $\left(\bar{p}_{1}, \bar{p}_{2}\right)$. We write $\tilde{l}_{2}=\frac{\bar{p}_{1} \cdot l_{2}}{m_{1}} \frac{\bar{p}_{1}}{m_{1}}-\left(k_{1} \cdot l_{2}\right) k_{1}=l_{2}^{0} \frac{\bar{p}_{1}}{m_{1}}+l_{2}^{1} k_{1}$. Using the Lorentz transformation

$$
\left(\begin{array}{l}
l_{2}^{0} \\
l_{2}^{1}
\end{array}\right)=\Lambda\left(\begin{array}{l}
L_{2}^{0} \\
L_{2}^{1}
\end{array}\right)=\left(\begin{array}{cc}
\sigma & \sqrt{\sigma^{2}-1} \\
\sqrt{\sigma^{2}-1} & \sigma
\end{array}\right)\left(\begin{array}{l}
L_{2}^{0} \\
L_{2}^{1}
\end{array}\right),
$$

we have $\bar{p}_{2} \cdot l_{2}=m_{2} L_{2}^{0}$ and $\bar{p}_{1} \cdot l_{2}=m_{1} \sigma L_{2}^{0}+m_{1} \sqrt{\sigma^{2}-1} L_{2}^{1}$. Similarly, we have $\bar{p}_{1} \cdot l_{1}=m_{1} l_{1}^{0}, \quad \bar{p}_{2} \cdot l_{1}=m_{2} \sigma l_{1}^{0}-$ $m_{2} \sqrt{\sigma^{2}-1} l_{1}^{1}$.

We have as well that

$$
\begin{aligned}
\left(l_{1}+l_{2}+u_{q}\right)^{2}= & \left(l_{1}+L_{2}+u_{q}\right)^{2} \\
& +2(1-\sigma)\left(k_{1} \cdot l_{1}\right)\left(k_{1} \cdot L_{2}\right),
\end{aligned}
$$

where we have used the conditions $\bar{p}_{1} \cdot l_{1}=\bar{p}_{2} \cdot l_{2}=0$ that are imposed by the delta functions.

The implies that the integral with the delta functions $\mathcal{D}$ can be written as

$$
\begin{aligned}
\mathcal{D}= & \frac{1}{m_{1}^{n_{1}+1} m_{2}^{n_{2}+1}\left(\sigma^{2}-1\right)^{\frac{n_{1}+n_{2}}{2}}} \int \frac{d^{D} l_{1} d^{D} L_{2}}{(2 \pi)^{2 D-2}} \frac{\delta\left(m_{1} l_{1}^{0}\right) \delta\left(m_{2} L_{2}^{0}\right)}{\left(-k_{1} \cdot l_{2} \pm i \varepsilon\right)^{n_{1}}\left(k_{1} \cdot l_{1} \pm i \varepsilon\right)^{n_{2}}\left(\left(u_{q}+l_{1}\right)^{2}\right)^{n_{3}}} \\
& \times \frac{1}{\left(\left(u_{q}+L_{2}\right)^{2}\right)^{n_{4}}\left(l_{1}^{2}\right)^{n_{5}}\left(L_{2}^{2}\right)^{n_{6}}\left(\left(l_{1}+L_{2}+u_{q}\right)^{2}+2(1-\sigma)\left(k_{1} \cdot l_{1}\right)\left(k_{1} \cdot L_{2}\right)\right)^{n_{7}}},
\end{aligned}
$$


which leads to the $D-1$ integrals

$$
\begin{aligned}
\mathcal{D}= & \frac{(-1)^{n_{1}}}{m_{1}^{n_{1}+1} m_{2}^{n_{2}+1}\left(\sigma^{2}-1\right)^{\frac{n_{1}+n_{2}}{2}}} \int \frac{d^{D-1} l_{1} d^{D-1} l_{2}}{(2 \pi)^{2 D-2}} \frac{1}{\left(k \cdot l_{2} \mp i \varepsilon\right)^{n_{1}}\left(k \cdot l_{1} \pm i \varepsilon\right)^{n_{2}}\left(\left(u_{q}+l_{1}\right)^{2}\right)^{n_{3}}} \\
& \times \frac{1}{\left(\left(u_{q}+l_{2}\right)^{2}\right)^{n_{4}}\left(l_{1}^{2}\right)^{n_{5}}\left(l_{2}^{2}\right)^{n_{6}}\left(\left(l_{1}+l_{2}+u_{q}\right)^{2}+2(1-\sigma)\left(k \cdot l_{1}\right)\left(k \cdot l_{2}\right)\right)^{n_{7}}},
\end{aligned}
$$

which we use to build the basis of master integrals (157) at two-loop order.

[1] B. P. Abbott et al. (LIGO Scientific and Virgo Collaborations), Observation of Gravitational Waves from a Binary Black Hole Merger, Phys. Rev. Lett. 116, 061102 (2016).

[2] B. P. Abbott et al. (LIGO Scientific and Virgo Collaborations), GW170817: Observation of Gravitational Waves from a Binary Neutron Star Inspiral, Phys. Rev. Lett. 119, 161101 (2017).

[3] T. Damour, Gravitational scattering, post-Minkowskian approximation and effective one-body theory, Phys. Rev. D 94, 104015 (2016).

[4] T. Damour, High-energy gravitational scattering and the general relativistic two-body problem, Phys. Rev. D 97, 044038 (2018).

[5] N.E. J. Bjerrum-Bohr, P. H. Damgaard, G. Festuccia, L. Planté, and P. Vanhove, General Relativity from Scattering Amplitudes, Phys. Rev. Lett. 121, 171601 (2018).

[6] C. Cheung, I. Z. Rothstein, and M. P. Solon, From Scattering Amplitudes to Classical Potentials in the Post-Minkowskian Expansion, Phys. Rev. Lett. 121, 251101 (2018).

[7] Z. Bern, C. Cheung, R. Roiban, C. H. Shen, M. P. Solon, and M. Zeng, Scattering Amplitudes and the Conservative Hamiltonian for Binary Systems at Third Post-Minkowskian Order, Phys. Rev. Lett. 122, 201603 (2019).

[8] Z. Bern, C. Cheung, R. Roiban, C. H. Shen, M. P. Solon, and $\mathrm{M}$. Zeng, Black hole binary dynamics from the double copy and effective theory, J. High Energy Phys. 10 (2019) 206.

[9] C. Cheung and M. P. Solon, Classical gravitational scattering at $\mathcal{O}\left(G^{3}\right)$ from Feynman diagrams, J. High Energy Phys. 06 (2020) 144.

[10] G. Kälin, Z. Liu, and R. A. Porto, Conservative Dynamics of Binary Systems to Third Post-Minkowskian Order from the Effective Field Theory Approach, Phys. Rev. Lett. 125, 261103 (2020).

[11] P. Di Vecchia, C. Heissenberg, R. Russo, and G. Veneziano, Universality of ultra-relativistic gravitational scattering, Phys. Lett. B 811, 135924 (2020).

[12] J. Parra-Martínez, M. S. Ruf, and M. Zeng, Extremal black hole scattering at $\mathcal{O}\left(G^{3}\right)$ : Graviton dominance, eikonal exponentiation, and differential equations, J. High Energy Phys. 11 (2020) 023.

[13] E. Herrmann, J. Parra-Martínez, M. S. Ruf, and M. Zeng, Radiative classical gravitational observables at $\mathcal{O}\left(G^{3}\right)$ from scattering amplitudes, arXiv:2104.03957.
[14] D. A. Kosower, B. Maybee, and D. O'Connell, Amplitudes, observables, and classical scattering, J. High Energy Phys. 02 (2019) 137.

[15] P. Di Vecchia, C. Heissenberg, R. Russo, and G. Veneziano, Radiation reaction from soft theorems, Phys. Lett. B 818, 136379 (2021).

[16] P. Di Vecchia, C. Heissenberg, R. Russo, and G. Veneziano, The eikonal approach to gravitational scattering and radiation at $\mathcal{O}\left(G^{3}\right)$, arXiv:2104.03256.

[17] D. A. Kosower, All order collinear behavior in gauge theories, Nucl. Phys. B552, 319 (1999).

[18] D. Bini, T. Damour, and A. Geralico, Sixth post-Newtonian local-in-time dynamics of binary systems, Phys. Rev. D 102, 024061 (2020).

[19] J. Blümlein, A. Maier, P. Marquard, and G. Schäfer, The 6th post-Newtonian potential terms at $O\left(G_{N}^{4}\right)$, Phys. Lett. B 816, 136260 (2021).

[20] T. Damour, Radiative contribution to classical gravitational scattering at the third order in $G$, Phys. Rev. D 102, 124008 (2020).

[21] Z. Bern, J. Parra-Martinez, R. Roiban, M. S. Ruf, C. H. Shen, M. P. Solon, and M. Zeng, Scattering amplitudes and conservative binary dynamics at $\mathcal{O}\left(G^{4}\right)$, Phys. Rev. Lett. 126, 171601 (2021).

[22] A. K. Collado, P. Di Vecchia, and R. Russo, Revisiting the second post-Minkowskian eikonal and the dynamics of binary black holes, Phys. Rev. D 100, 066028 (2019).

[23] M.E. Peskin and D. V. Schroeder, An Introduction to Quantum Field Theory (Perseus book, 1995).

[24] V. A. Smirnov, Evaluating Feynman Integrals (SpringerVerlag Berlin Heidelberg, 2004).

[25] R. N. Lee, Litered 1.4: A powerful tool for reduction of multiloop integrals, J. Phys. Conf. Ser. 523, 012059 (2014).

[26] N. E. J. Bjerrum-Bohr, J.F. Donoghue, and P. Vanhove, On-shell techniques and universal results in quantum gravity, J. High Energy Phys. 02 (2014) 111.

[27] A. Cristofoli, P. H. Damgaard, P. Di Vecchiam, and C. Heissenberg, Second-order post-Minkowskian scattering in arbitrary dimensions, J. High Energy Phys. 07 (2020) 122.

[28] M. B. Green, J. H. Schwarz, and E. Witten, Superstring Theory, Cambridge Monographs on Mathematical Physics, Vol. 1 (Cambridge University Press, Cambridge, England, 1987). 
[29] M. B. Green, J. H. Schwarz, and E. Witten, Superstring Theory, Cambridge Monographs on Mathematical Physics, Vol. 2 (Cambridge University Press, Cambridge, England, 1987).

[30] S. Caron-Huot and Z. Zahraee, Integrability of black hole orbits in maximal supergravity, J. High Energy Phys. 07 (2019) 179.

[31] M. B. Green, J. H. Schwarz, and L. Brink, $\mathcal{N}=4$ YangMills and $\mathcal{N}=8$ supergravity as limits of string theories, Nucl. Phys. B198, 474 (1982).

[32] Z. Bern, L. J. Dixon, D. C. Dunbar, M. Perelstein, and J.S. Rozowsky, On the relationship between Yang-Mills theory and gravity and its implication for ultraviolet divergences, Nucl. Phys. B530, 401 (1998).

[33] P. Di Vecchia, S. G. Naculich, R. Russo, G. Veneziano, and C. D. White, A tale of two exponentiations in $\mathcal{N}=8$ supergravity at subleading level, J. High Energy Phys. 03 (2020) 173.
[34] N. E. J. Bjerrum-Bohr, J. F. Donoghue, B. R. Holstein, L. Planté, and P. Vanhove, Bending of Light in Quantum Gravity, Phys. Rev. Lett. 114, 061301 (2015).

[35] Z. Bern, H. Ita, J. Parra-Martinez, and M. S. Ruf, Universality in the Classical Limit of Massless Gravitational Scattering, Phys. Rev. Lett. 125, 031601 (2020).

[36] N. E. J. Bjerrum-Bohr and P. Vanhove, Absence of triangles in maximal supergravity amplitudes, J. High Energy Phys. 10 (2008) 006.

[37] J. M. Henn, Lectures on differential equations for Feynman integrals, J. Phys. A 48, 153001 (2015).

[38] S. Borowka, G. Heinrich, S. Jahn, S. P. Jones, M. Kerner, J. Schlenk, and T. Zirke, Pysecdec: A toolbox for the numerical evaluation of multi-scale integrals, Comput. Phys. Commun. 222, 313 (2018).

[39] J. Dieudonné, Calcul Infinitésimal (Nouveau tirage édition, Hermann, 1997).

[40] D. N. Kabat and M. Ortiz, Eikonal quantum gravity and Planckian scattering, Nucl. Phys. B388, 570 (1992). 